\title{
The Arabidopsis condensin CAP-D subunits arrange interphase chromatin
}

\section{Running title: Arabidopsis CAP-D proteins}

Celia Municio ${ }^{1}$, Wojciech Antosz ${ }^{2}$, Klaus D. Grasser ${ }^{2}$, Etienne Kornobis ${ }^{3,4}$, Michiel Van Bel ${ }^{5}$, Ignacio Eguinoa $^{5}$, Frederik Coppens ${ }^{5}$, Andrea Bräutigam ${ }^{1}$, Inna Lermontova ${ }^{1}$, Astrid.Bruckmann ${ }^{2}$, Andreas Houben ${ }^{1}$, Veit Schubert ${ }^{1 *}$

${ }^{1}$ Leibniz Institute of Plant Genetics and Crop Plant Research (IPK) Gatersleben, Corrensstraße 3, D06466 Seeland, Germany

${ }^{2}$ Cell Biology and Plant Biochemistry, Biochemistry Center, University of Regensburg, Universitätsstraße 31, D-93053 Regensburg, Germany

${ }^{3}$ Biomics, C2RT, Institut Pasteur, Paris, France

${ }^{4}$ Hub Bioinformatique et Biostatistique, Département de Biologie Computationnelle - USR 3756

CNRS, Institut Pasteur, Paris, France

${ }^{5}$ VIB-UGent Center for Plant Systems Biology, Technologiepark 71, 9052 Gent, Belgium

*To whom correspondence should be addressed: schubertv@ipk-gatersleben.de

Telephone: $\quad+49-39482-5212$ 


\section{SUMMARY}

Condensins are best known for their role in shaping chromosomes. However, other functions as organizing interphase chromatin and transcriptional control have been reported in yeasts and animals. Yeasts encode one condensin complex, while higher eukaryotes have two of them (condensin I and II). Both, condensin I and II, are conserved in Arabidopsis thaliana, but so far little is known about their function. Here we show that the A. thaliana CAP-D2 (condensin I) and CAP-D3 (condensin II) subunits are highly expressed in mitotically active tissues. In silico and pull-down experiments indicate that both CAP-D proteins interact with the other condensin I and II subunits. Our data suggest that the expression, localization and composition of the condensin complexes in A. thaliana are similar as in other higher eukaryotes. Previous experiments showed that the lack of A. thaliana CAP-D3 leads to centromere association during interphase. To study the function of CAP-D3 in chromatin organization more in detail we compared the nuclear distribution of rDNA, of centromeric chromocenters and of different epigenetic marks, as well as the nuclear size between wild-type and cap- $d 3$ mutants. In these mutants an association of heterochromatic sequences occurs, but nuclear size and the general methylation and acetylation patterns remain unchanged. In addition, transcriptome analyses revealed a moderate influence of CAP-D3 on general transcription, but a stronger one on transcription of stress-related genes. We propose a model for the CAP-D3 function during interphase, where CAP-D3 localizes in euchromatin loops to stiff them, and consequently separates centromeric regions and $45 \mathrm{~S}$ rDNA repeats.

\section{Keywords}

Arabidopsis thaliana, chromatin organization, chromosomes, condensin, interphase nuclei, SMC proteins

\section{INTRODUCTION}

The spatial genome arrangement is important to regulate the access of proteins to DNA (Gibcus and Dekker, 2013), since the folding of chromatin allows or impedes interactions between distinct loci and their regulatory sequences (Doğan and Liu, 2018; Robson et al., 2019; Stam et al., 2019; Szabo et al., 2019). Thus, a better knowledge of the nuclear organization during interphase could help to understand processes like replication, DNA repair, recombination and transcription. During interphase, in A. thaliana (Pecinka et al., 2004) as well as in other higher eukaryotes, the chromosomes occupy discrete regions called chromosome territories. Although this higher order chromatin arrangements is highly conserved, species-specific structural and functional features of nuclear organization exist in metazoans and protists (Cremer and Cremer, 2010; Cremer et al., 2018). In contrast to mammals (Boyle et al., 2001; Mayer et al., 2005) and birds (Habermann et al., 2001), A. thaliana chromosome territories prefer no particular position within the nucleus (Pecinka et al., 2004).

Chromocenters are chromatin structures intensely stained by DNA-specific dyes and represent condensed heterochromatin regions in interphase nuclei (Jost et al., 2012). In A. thaliana chromocenters incarnate centromeric and pericentromeric heterochromatin located near the nuclear periphery and the nucleolus (Fransz et al., 2002; Schubert et al., 2012). For the maintenance of chromocenters different proteins related to methylation, ATPases and nuclear periphery components were described in A. thaliana (Soppe et al., 2002; Moissiard et al., 2012; Wang et al., 2013; Poulet et al., 2017).

To explain the organization of chromosome territories in A. thaliana interphase nuclei, a rosette model was proposed (Fransz et al., 2002). Based on cytological observation and later support by computer simulations (de Nooijer et al., 2009) and Hi-C data (Feng et al., 2014; Liu et al., 2016), this model assumes that the chromosomes are organized as chromatin loops emanating from the chromocenters.

Structural Maintenance of Chromosomes (SMC) complexes are present in prokaryotes and eukaryotes (Cobbe and Heck, 2004). They are essential for chromatin organization and dynamics, gene regulation and DNA repair. In eukaryotes six conserved SMC subunits form the core of three different complexes: cohesin, involved in sister chromatids cohesion and interphase chromatin arrangement; condensin, involved in mitotic and meiotic chromosome organization (van Ruiten and Rowland, 2018; Skibbens, 2019); and the SMC5/SMC6 complex, mainly involved in DNA repair and replication 
(Jeppsson et al., 2014). Animals have two condensin complexes, condensin I and II (Ono et al., 2003). In yeasts, only one condensin complex analogous to animal condensin I is present (Freeman et al., 2000; Hirano, 2012a). Condensin I and II share a core formed by SMC2 and SMC4 and differ in the associated proteins, which are in condensin I CAP-H, CAP-D2 and CAP-G, and in condensin II CAPH2, CAP-D3 and CAP-G2 (Ono et al., 2003; Hirano, 2012a). This composition is conserved in higher eukaryotes, although in Drosophila the subunit CAP-G2 of condensin II has not been detected (Herzog et al., 2013). As proposed for A. thaliana (Fig. 1), plants apparently have condensin I and II.

Condensins have been widely studied in human, animals and yeast for their role in shaping chromosomes. Together with topoisomerase II condensins form a scaffold within human somatic metaphase chromatids (Maeshima and Laemmli, 2003). Depletion of condensin I causes short fuzzy metaphase chromosomes while the depletion of condensin II causes long and curly chromosomes (Ono et al., 2003; Green et al., 2012). Besides aberrant chromosome morphologies, chromosomes lacking several condensin subunits show anaphase bridges and other segregation defects (Freeman et al., 2000; Hudson et al., 2003; Ono et al., 2003, 2004; Hirota et al., 2004; Savvidou et al., 2005; Gerlich et al., 2006; Hartl et al., 2008). Both complexes may form DNA loops resulting in chromosome compaction (Elbatsh et al., 2019; Gibcus et al., 2018; van Ruiten and Rowland, 2018; Walther et al., 2018).

Condensin I and II complexes show a distinct subcellular localization during the mammalian cell cycle. In human and rat during interphase, condensin I occurs in the cytoplasm, condensin II in the nucleus (Hirota et al., 2004; Ono et al., 2004). During mitosis, condensin I and II localize along the chromosome arms in an alternate fashion, and both are enriched at the centromeres (Ono et al., 2003; Ono et al., 2004; Savvidou et al., 2005). In addition to the canonical role in metaphase chromosome formation, condensins are also involved in gene expression and chromatin organization during interphase (Wallace and Bosco, 2013; Wallace et al., 2015). In mouse and human, condensin II localizes at the promoters of active genes and is required for normal gene expression (Dowen et al., 2013; Yuen et al., 2017;). In Drosophila, CAP-D3 together with the RetinoBlastoma protein RBF1, regulates gene clusters involved in tissue-specific functions (Longworth et al., 2012), and condensin II promotes the formation of chromosome territories and keeps repetitive sequence clusters apart from each other (Hartl et al., 2008; Bauer et al., 2012; Hirano, 2012b; Rosin et al., 2018).

Also A. thaliana posseses the components for both condensin complexes (Schubert, 2009; Smith et al., 2014). In contrast to other organisms, A. thaliana has two SMC2 homologs, SMC2A and SMC2B with redundant functions (Siddiqui et al., 2003)(Fig. 1). As in other species, SMC4, CAP-H and CAP-H2 are present within chromosomes and are required for normal metaphase chromosome compaction (Fujimoto et al., 2005; Smith et al., 2014). During interphase, CAP-H is present in the cytoplasm of protoplasts while the condensin II subunits CAP-H2 and CAP-D3 were detected in the nucleolus and euchromatin, respectively (Fujimoto et al., 2005; Schubert et al., 2013). CAP-D2 and CAP-D3 prevent the association of centromeres and induce chromatin compaction (Schubert et al., 2013). The requirement of the condensin II-specific subunits CAP-H2 and CAP-G2 for keeping centromeres apart has been confirmed by Sakamoto et al. (2019). In addition, these authors showed that condensin II is necessary for the correct spatial arrangement between centromeres and rDNA arrays.

Condensins are highly conserved, but have not been studied extensively in plants. Here we analyze the A. thaliana CAP-D2 and CAP-D3 condensin subunit expression patterns, their cellular localization and interaction with other condensin subunits and additional proteins for better understanding their functions. We demonstrate that also A. thaliana forms specific condensin I and II complexes, and show that CAP-D3 mediates the spatial separation of chromocenters, without altering the global methylation pattern and nuclear ultrastructure. Finally, we suggest a model explaining the action of CAP-D3 to prevent the association of chromocenters.

\section{RESULTS}

\section{$C A P-D 2$ and $C A P-D 3$ are highly expressed in meristematic tissues}

Based on in silico analysis using the Arabidopsis eFP Browser (Winter et al., 2007), A. thaliana CAPD2 (At3g57060) and CAP-D3 (At4g15890) have a similar expression pattern. Both proteins are highly expressed in the shoot apex, roots, flower buds and vegetative rosette leaves. Their expression is lower in cotyledons, rosette leaves after bolting, mature flowers, siliques and embryos (Figure S1). To 
corroborate the in silico data we assessed the transcription of both genes in seedlings, mature rosette leaves, roots and flower buds by quantitative real-time RT-PCR. The highest transcription of both genes was observed in flower buds, the lowest in seedlings. The transcription level of $C A P-D 2$ is 25.6 , 14.8 and 3.5 times higher in flower buds, roots and leaves, respectively, than in seedlings. Similarly, the CAP-D3 transcription is 18.3, 9.4 and 4.4 times higher in flower buds, roots and leaves respectively, than in seedlings (Fig. 2).

The activity of the $C A P-D 2$ and $C A P-D 3$ promoters was evaluated in A. thaliana transgenic lines expressing different versions of the promoters fused to the $\beta$-glucuronidase (GUS) reporter gene (Fig. $3 a)$. Six presumed promoters of different length were analyzed for $C A P-D 2$ and two for $C A P-D 3$. The promoter region of $C A P-D 2$ contains two putative E2F binding sites at -345 bp and -114 bp upstream from the start of the gene (Schubert et al., 2013). Two promoter lengths were analyzed: a promoter that comprises $1156 \mathrm{bp}$ upstream of the start of CAP-D2 (Pro4), and a short promoter of $391 \mathrm{bp}$ (Pro7). In addition, promoter proximal introns can enhance the expression of a gene by a mechanism known as Intron-Mediated Enhancement (IME) (Rose et al., 2008). The putative enhancing ability of $C A P-D 2$ introns was analyzed in silico with the web tool IMEter (Parra et al., 2011). The IMEter score is positively correlated to the enhancing ability of an intron. For $C A P-D 2$ the two first introns have positive IMEter scores of 12.13 and 2.36, respectively. Thus, it is likely that they enhance expression. These introns were included in the analysis in combination with the long and short promoters of $C A P$ D2: Pro5 (long promoter) and Pro8 (short promoter) include Intron1; and Pro6 (long promoter) and Pro9 (short promoter) include both Intron1 and Intron2. The promoter region of CAP-D3 contains also two putative E2F binding sites at $-397 \mathrm{bp}$ and $-84 \mathrm{bp}$ (Schubert et al., 2013). However, the IMEter scores of the first two CAP-D3 introns were negative, -13.20 and -5.88 respectively. Thus, it is unlikely that they enhance expression. Therefore, for $C A P-D 3$ the introns were not considered and only a long promoter at -1318 bp (Pro10) and a short promoter at -474 bp (Pro11) from the start of the gene were analyzed.

T1 transgenic plants with the different versions of $C A P-D 2$ and $C A P-D 3$ promoters were stained for GUS analysis (Fig. 3b). Only for Pro4 no positive plants could be isolated. The CAP-D2 promoter version Pro5 ( $\mathrm{n}=7$ ) was active in stipules (small organs at the base of the leaves), leaf vascular tissue and root tip meristems. Pro6 ( $n=6)$ had weak activity in root tips. All Pro7 plants $(n=21)$ showed GUSstaining in leaf vascular tissue and root tips, and 16 plants also in stipules. All Pro8 plants $(n=23)$ presented GUS activity in the apical meristem and root tips, and 16 of them also in leaf vascular tissue. Pro9 ( $\mathrm{n}=5)$ showed activity in roots, and 3 plants also weakly in the apical meristem (Fig. 1d). Therefore, all $C A P-D 2$ promoter versions were active in root tips, but the staining was stronger in the short promoter versions (Pro7, Pro8 and Pro9) than in the long ones (Pro5 and Pro6). In addition, the $C A P-D 2$ short promoters showed an activity in the apical meristem and versions that included the second intron (Pro6 and Pro9) lost the staining in the leaf vascular tissue. CAP-D3 Pro10 showed no activity, and for Pro11 ( $\mathrm{n}=8)$, the plants showed activity in the apical meristem and root tips. For both, $C A P-D 2$ and $C A P-D 3$, the expression can be driven more effectively by the short promoter, which contains the E2F sites.

Taken together quantitative real-time RT-PCR and GUS activity staining demonstrated that, CAP-D2 and $C A P-D 3$ are highly expressed in meristematic tissues (root tip meristem, flower buds, apical meristem) and young leaves and less expressed in mature leaves. The low transcription observed in seedlings could be due to a low amount of meristematic tissue in the sample since just one-week old seedlings were used for RNA isolation.

\section{CAP-D2 and CAP-D3 interact with the other condensin subunits in specific complexes}

CAP-D2 and CAP-D3 are specific components of condensin I and II complexes, respectively. The presence of CAP-D2 and CAP-D3 as well as the other condensin complex subunits in A. thaliana was previously confirmed (Smith et al., 2014), but whether the complexes are formed by the same subunits as in non-plant species is unknown. To predict a composition of each complex we identified putative interactors of CAP-D2 (Figure S2a) and CAP-D3 (Figure S2b) in silico using the STRING program (http://string-db.org/; Szklarczyk et al., 2019). At the high score of $>0.90$ the following proteins: SMC2A (At5g62410), SMC2B (At3G47460) and SMC4 (At5g48600) were identified in interaction networks of both CAP-D2 and CAP-D3, while CAP-G (At5g37630) and CAP-H (At2g32590) were found as interactors of CAP-D2, and CAP-G2 (At1g64960) and CAP-H2 (At3g16730) as specific interactors of CAP-D3, respectively. Due to the presence of SMC2A, SMC2B and SMC4 in both 
interaction networks they may be involved in the formation of condensin I as well as of condensin II. In silico analysis using the STRING program identified besides cohesin subunits also SMC5/6 complex subunits as CAP-D2 and CAP-D3 interacting partners (Zelkowski et al., 2019).

To confirm these in silico results and to determine the composition of each complex experimentally, CAP-D2 and CAP-D3 were fused to a GS-tag, and affinity-purified from A. thaliana PSB-D suspension cultured cells (Figure S3). The proteins co-purifiying with CAP-D2-GS and CAP-D3-GS were identified by mass spectrometry. The putative subunits of the condensin I complex, SMC2A, SMC2B, SMC4, CAP-H and CAP-G, were detected with high scores in the CAP-D2-GS eluates of three affinity purifications performed. Similarly, the putative subunits of the condensin II complex, SMC2A, SMC4, CAP-H2 and CAP-G2, were detected in the three affinity purifications performed for CAP-D3-GS and SMC2B in two of the affinity purifications (Table 1, Fig. 1). Like in the in silico analysis, CAP-H, CAP-G and CAP-H2, CAP-G2 were identified as specific components of the condensin I and condensin II complexes, respectively, while SMC2A, SMC2B and SMC4 coprecipitated with both CAP-D2 and CAP-D3.The results indicate that $A$. thaliana, similar as mammals, chicken and C. elegans (Hirano, 2012a; Onn et al., 2007), comprises specific condensin I and II complexes. Interestingly, in addition to SMC4, both SMC2A and SMC2B may be involved in the formation of both condensin complexes.

Among the proteins which co-purified with CAP-D2 (Table S1), other proteins such as the cohesin complex subunit SMC3 were identified. Additionally, the chromatin remodeling factors CHR17 and CHR19; CUL1, a subunit of the SCF ubiquitin ligase complex; HDC1, a histone deacetylase and ELO3, a histone acetyltransferase from the elongator complex were found. Among the proteins copurifying with CAP-D3 (Table S2) were two nucleosome assembly proteins (NAP); CSN1, a subunit of the COP9 signalosome (CSN); the helicase BRAHMA; ELO3, from the elongator complex, and NERD, involved in DNA methylation.

The results indicate that both $A$. thaliana Cap-D genes are highly conserved, and that the corresponding proteins may act in combination with other condensin complex components, as well as with cohesin and SMC5/6 subunits.

\section{Condensin I subunits are localized within nuclei and cytoplasm}

Previously, A. thaliana protoplasts have been used to examine the localization of the condensin subunits CAP-H and CAP-H2 (Fujimoto et al., 2005). Therefore, we expressed transiently the coding region of CAP-D2 fused to EYFP (35S::CAP-D2_EYFPc) in A. thaliana mesophyll protoplasts. To visualize EYFP, the protoplasts were immunolabeled with anti-GFP antibodies. We identified CAPD2 in the cytoplasm and the DAPI-counterstained nucleus (Fig. 4a). In the cytoplasm GFP-negative, but DAPI-positive round chloroplasts were also visible. The free EYFP of the positive control also localized in the cytoplasm and nucleus. Western blot analysis of CAP-D2_EYFPc transformed protoplasts confirmed that the CAP-D2_EYFP protein was intact, and that the visible localization corresponds to the fusion protein (187 kDa), and not to free EYFP (27 kDa) (Fig. 4b). The condensin I subunits, CAP-H and CAP-G fused to EYFP localized also in the cytoplasm and the nucleus (Figure S4). Similarly, transient transformation of $N$. benthamiana leaves revealed the localization of CAPD2, CAP-H and CAP-G EYFP-fusion proteins in cytoplasm and nuclei too (Figure S5).

Anti-CAP-D2 Western blot antibodies were generated against a recombinant protein containing the last 501 amino acids of CAP-D2 (Figure S6). The CAP-D2 antiserum can detect amounts as low as 1 ng of the recombinant protein (Figure S7). The CAP-D2 antiserum detects the CAP-D2 fusion protein from protoplasts (Fig. 4b), but not the CAP-D2 protein from wild-type leaves (data not shown). This may be due to a lower amount of the target protein in leaves compared to that in protoplasts. In protoplast overexpression of CAP-D2 occurred since the reporter construct is under control of the $35 \mathrm{~S}$ promoter.

In order to localize CAP-D2 and CAP-D3 proteins in planta, A. thaliana wild-type plants were transformed with constructs containing the coding region of either gene fused at its C-terminus to enhanced yellow fluorescence protein (EYFP) under the control of the $35 \mathrm{~S}$ promoter $(35 \mathrm{~S}: \mathrm{CAP}$ D2_EYFPC and 35S::CAP-D3_EYFPC). In both cases, the detection of the proteins in vivo or by immunolocalization with anti-GFP antibodies (also detecting EYFP) was not possible. The same negative result was obtained by reporter constructs with EYPF fused at the N-terminus (35S::CAP-D2_EYFPn and 35S::CAP-D3_EYFPn). 


\section{CAP-D3 organizes chromatin during interphase}

The involvement of A. thaliana CAP-D3 in compacting chromosome territories (CT) and keeping centromeres apart at interphase has been previously described by Schubert et al. (2013). In Drosophila, CAP-D3 is also involved in the formation of compact chromosome territories (Hartl et al., 2008). To further study the involvement of CAP-D3 in chromatin organization we used two cap-d3 mutants described previously, Cap-D3 SAIL_826_B06 and Cap-D3 SALK_094776 (Schubert et al., 2013) (Fig. 5a,b). To confirm the centromeric clustering and CT dispersion phenotypes in both mutants, a FISH experiment on flow-sorted 4C nuclei was performed with probes specific for the centromere repeat pAL and the chromosome 1 arm territory bottom (CT1B) (Fig. 5c, d). In addition to the number of centromeric pAL signals per nucleus, the areas of the CT1B signals and the nucleus were measured. The median area size of the CT1B signals was 3.9, 4.7 and $4.7 \mu \mathrm{m}^{2}$ for cap- $d 3 \mathrm{SAIL}$, cap- $d 3 S A L K$ and wild-type, respectively (Fig. 5e). No significant differences were found. Thus, we could not confirm the CT dispersion phenotype of the cap- $d 3$ mutants described in Schubert et al. (2013). In addition, no significant differences were found in the nuclear area size between the cap- $d 3$ mutants and wild-type plants (Fig. 5e).

On the other hand, we could confirm the centromere-association phenotype. In both cap- $d 3$ mutants the nuclei showed a lower number of centromeric pAL signal clusters than wild-type (Fig. 5d). Around $80 \%$ of the cap- $d 3$ mutant nuclei showed less than six pAL signals, while in wild-type only $12 \%$ of nuclei had less than six pAL signals (Fig. 5f). To verify that the mutation in the CAP-D3 gene is indeed responsible for the centromeric clustering, a complementation experiment was carried out. cap-d3 SALK mutant plants were transformed with CAP-D3_EYFPc constructs, containing the coding region of $C A P-D 3$ fused to EYFP under the control of the $35 \mathrm{~S}$ promoter. The centromeric association phenotype was evaluated in cap-d3 SALK complemented plants by FISH and compared with the capd3 SALK mutants and wild-type. Only $15 \%$ of the complemented nuclei showed less than six centromeric signal clusters, which is similar as the wild-type association levels (Fig. 5g). This confirms that CAP-D3 is responsible for the centromere association in the mutants.

Beside centromeres, in A. thaliana, the 45S and 5S rDNAs are heterochromatin-associated sequences. In nuclei of differentiated cells, $45 \mathrm{~S}$ rDNA containing nucleolar organizing regions tend to associate, but the 5S rDNA loci are often separated (Berr and Schubert, 2007). To examine whether CAP-D3 affects in general the organization of heterochromatin, the distribution of the $45 \mathrm{~S}$ and $5 \mathrm{~S}$ rDNA loci was analyzed by FISH in both cap- $d 3$ mutants (Fig. 6a). The majority of $45 \mathrm{~S}$ rDNA signals is shifted from three signals in wild-type to two signals in the mutants (Fig. 6b). No difference was observed with regard to 5S rDNA since over 70\% of the nuclei showed between six and ten signals in the cap$d 3$ mutant and wild-type plants (Fig. 6c). Thus, the cap- $d 3$ mutants present a higher association of the chromosomal 45S rDNA regions than wild-type, but the number of 5S rDNA signals remains unaffected.

A. thaliana centromeres are positioned at the nuclear periphery (Fransz et al., 2002; Fang and Spector, 2005). To test whether the centromere position is influenced by the cap-d3 mutations nuclei were embedded in acrylamide to preserve their 3D structure followed by FISH (3D-FISH) with the centromeric pAL repeats. For each genotype, cap-d3 SAIL, cap-d3 SALK and wild-type, 10 nuclei were analyzed. Optical sections (3D-SIM stacks) were acquired for each nucleus, and the centromere positions were analyzed in the ZEN software tool 'ortho view' (Fig. 6d). In all the cases, the centromeres were localized at the periphery of the nucleus, even when centromere clustering was present in the cap- $d 3$ mutants. Consequently, no deviation in peripheral centromere positioning in wild-type and the cap- $d 3$ mutants was observed.

\section{CAP-D3 does not effect the nuclear distribution of histone marks}

DNA can be methylated at cytosine as 5-methyl-cytosine $(5 \mathrm{mC})$. The methylation of DNA is associated with heterochromatin formation and consequently, it has been found in the chromocenters of A. thaliana (Fransz et al., 2002). Mouse embryonic stem cells depleted of condensin show a reduction of 5mC (Fazzio and Panning, 2010). In order to test whether such an effect can also be observed in plants, the distribution of methylated DNA in cap- $d 3$ mutants was compared to wild-type by immunodetection of $5 \mathrm{mC}$-specific sites. In both cap-d3 mutants and wild-type the $5 \mathrm{mC}$ signals were similarly chromocenter-localized (Fig. 7a). The A. thaliana centromeric repeats are highly methylated in a CpG context (Martinez-Zapater et al., 1986). The use of methylation sensitive 
enzymes and Southern blot hybridization allowed a more precise determination of the relative DNA methylation level of the centromeric repeats. HpaII and its isoschizomer MspI cleave the same CCGG sequence, but HpaII is methylation sensitive while $M s p I$ is not. In wild-type, the centromeric repeats are highly methylated and are thus digestible by $M s p I$ (Fig. 7b). The ladder-like pattern corresponds to the monomer, dimer, trimer and higher orders of centromeric repeats. As expected, HpaII does not cut in wild-type DNA. In both cap- $d 3$ mutants, the hybridization pattern is similar to wild-type. Thus, the relative level of CCGG methylation is not altered in the cap- $d 3$ mutants (Fig. 7b).

CAP-D3 prevents the clustering of heterochromatin, but the CAP-D3 protein itself localizes in euchromatic regions during interphase. Both types of chromatin are characterized by specific posttranslational histone modifications marks (Fuchs et al., 2006). To evaluate a possible functional association between histone modifications and CAP-D3 functions, the global distribution patterns of different histone marks were compared between the cap- $d 3$ mutants and wild-type. Specific marks for heterochromatin (histone H3K9me1, H3K9me2) and euchromatin (histone H3K4me3, H3K27me3) were tested by indirect immunostaining. In addition, the $\mathrm{H} 3$ acetylation marks $\mathrm{H} 3 \mathrm{~K} 9 \mathrm{ac}, \mathrm{H} 3 \mathrm{~K} 14 \mathrm{ac}$, and $\mathrm{H} 3 \mathrm{~K} 18 \mathrm{ac}$ as well as $\mathrm{H} 3 \mathrm{~K} 9+14+18+23+27 \mathrm{ac}$ were evaluated. Histone acetylation relaxes chromatin allowing different protein complexes to access DNA (Wang et al., 2014). Thus, histone acetylation is associated with transcription, and hypoacetylation with transcriptional repression. In flow-sorted 4C wild-type nuclei, $\mathrm{H} 3 \mathrm{~K} 4 \mathrm{me} 3$ localizes in euchromatin and it is absent from chromocenters and the nucleolus. In cap- $d 3$ mutants the localization is identical. H3K9me1 is a heterochromatin-specific histone modification that localizes in the chromocenters in both cap-d3 mutants and wild-type. Finally, the acetylation mark H3K14ac localizes mainly in euchromatin (transcriptionally active chromatin) of wild-type nuclei, but also in the mutants (Fig. 7c). The other histone modifications tested (H3K27me3, H3K9me2, H3K9ac, H3K18ac and H3K14+18+23+27ac) followed also the same pattern in wild-type and the cap- $d 3$ mutants (Figure S8). Thus, we did not detect obvious differences in the (sub-)nuclear distribution patterns of the different histone marks between wild-type and the capd3 mutants.

\section{CAP-D3 moderately affects transcription}

To assess if the increased clustering of the centromeric interphase chromatin in the cap-d3 mutants affects gene transcription, the transcriptome of both cap- $d 3$ mutants was compared to wild-type. RNA-sequencing was performed in 4 samples (pooled 4 weeks-old plantlets) for each genotype. After differential expression analysis, we could observe alterations between the cap- $d 3$ mutants and wildtype transcriptomes. The genes with at least 2 -fold change transcription and a pAdj $\leq 0.05$ were considered as differentially expressed genes (DEG) between two genotypes (Fig. 8a). The smallest difference was observed between cap-d3 SAIL vs. cap-d3 SALK (74 DEG), and the highest between cap-d3 SAIL vs. wild-type (398 DEG). cap-d3 SALK vs. wild-type was intermediate (97 DEG)(Fig. $8 \mathrm{~b})$. Both cap- $d 3$ mutants show centromere and $45 \mathrm{~S}$ rDNA clustering, but cap-d3 SAIL plants showed additional growth defects that are absent in cap-d3 SALK plants. To separate the individual effect of each allele, in further analysis only the DEG shared by both mutants when compared to wild-type were considered. These 83 genes, common to the cap- $d 3$ mutation independently of the specific alleles, are subsequently referred to as "cap-d3 DEG" (Fig. 8b and Table S3). These genes are distributed along all chromosome arms (Fig. 8c). According to their Gene Ontology (GO) enrichment, the cap- $d 3$ DEGs are mainly involved in transcription, particularly in biological processes affecting the response to water, stimuli and stress (Table 2). In agreement with their role in transcription, 13 out of the 83 cap- $d 3$ DEG are transcription factors (Table S3). We conclude that the influence of CAP-D3 directly on transcription is moderate. However, the DEG involvement in plant response to stress, and the high proportion of transcription factors indicate that CAP-D3 may influence transcription indirectly.

\section{DISCUSSION}

\section{Arabidopsis CAP-D proteins are expressed in meristematic tissues in a cell cycle-dependent manner}

$C A P-D 2$ and $C A P-D 3$ are highly expressed in meristems and cell cycle active tissues (flower buds, roots), but weaker in non-cycling tissues (mature leaves). Similarly, the condensin subunit genes $C A P$ - 
$H$ and SMC2 are highly expressed in dividing tissues (Fujimoto et al., 2005; Liu et al., 2002; Siddiqui et al., 2003). Sequences of $391 \mathrm{bp}$ or $474 \mathrm{bp}$ upstream of the start of CAP-D2 or CAP-D3, respectively, are sufficient to act as promoters. Longer fragments (>1000 bp) do not improve the expression of the reporter gene. Interestingly, the $C A P-D 2$ and $C A P-D 3$ promoters regions contain two previously predicted E2F binding sites (Schubert et al., 2013). E2F is a transcriptional activator of genes important for cell cycle progression. Together with retinoblastoma-related protein (RBR) and a dimerization partner, they control the transition from G1 to S phase. E2F sites are also present in the A. thaliana SMC2 promoter (Siddiqui et al., 2003). In mouse, CNAP1 (CAP-D2) is also a target of E2F (Verlinden et al., 2005). Considering the expression patterns, the promoter features and the comparison with other organism, it is plausible that the transcription of A. thaliana CAP-D2 and CAP$D 3$ is cell cycle-regulated.

Introns, when affecting the expression of a gene, often enhance its expression by increasing the transcript amount or by inducing the expression in specific tissues (Rose et al., 2008; Parra et al., 2011; Heckmann et al., 2011). Nonetheless, the second intron of CAP-D2 could have intragenic regulatory sequences repressing the expression in non-dividing tissues. This is supported herein by the loss of GUS reporter expression in leaves of the Pro6 and Pro9 transgenic plants compared with Pro5, Pro7 and Pro8 plants, which do not carry the second intron. Moreover, our quantitative RT-PCR results showed low transcription of CAP-D2 in leaves. The second intron of the AGAMOUS gene is also responsible to inhibit expression in vegetative tissues, and drives its correct expression in flowers (Sieburth and Meyerowitz, 1997).

\section{The subunit composition of Arabidopsis condensin I and II is similar as in other eukaryotes}

Protein immunoprecipitation (IP) from flower bud extracts confirmed already the presence of the subunits for condensin I and condensin II in A. thaliana (Smith et al., 2014). Nonetheless, these IPs were performed with anti-SMC4, which would target both condensin complexes, and therefore could not determine the exact composition of condensin I and II. Our data based on affinity purification combined with mass spectrometry support that in A. thaliana both condensin complexes are present, and that their subunit composition is identical to those of other organisms (Hirano, 2012a). Notably, A.thaliana is the only species in which two SMC2 homologs have been predicted and described (Cobbe and Heck, 2004; Siddiqui et al., 2003). Both, SMC2A and SMC2B can be active, but SMC2A accounts for most of the SMC2 transcript pool (Siddiqui et al., 2003). Both SMC2A and SMC2B interact with the other condensin subunits in vegetative and somatic tissues (Smith et al., 2014; this study).

In human cells and Drosophila, CAP-D3 interacts with RBR and promotes the correct chromosomal localization of condensin II (Longworth et al., 2008). In A. thaliana, this interaction is likely not conserved, since we could not detect RBR among the proteins that co-purified with CAP-D3. In human, Cdc20, a component of the anaphase-promoting complex E3 ubiquitin ligase, interacts and regulates CAP-H2 (Kagami et al., 2017). In Drosophila, CAP-H2 also interacts and is regulated by the Skp cullin-F-box SCF ${ }^{\text {Slimb }}$ (Buster et al., 2013), an E3 ubiquitin ligase regulated by CSN (Hotton and Callis, 2008). In our affinity purifications, we also detected components of the ubiquitin-26S proteasome pathway. CULLIN 1 co-purified with CAP-D2 and CSN1 with CAP-D3 in all replicates. CSN3 and CSN4 also co-purified with CAP-D3 in the three triplicates but also in 3 out of 115 of the non-specific proteins affinity purifications (data not shown). CULLIN1 and CULLIN3 were present in two of the CAP-D3 triplicates. These data suggest that in A. thaliana, ubiquitination could be involved in the regulation of the condensins.

A screen for functional partners of condensin in yeast identified, among others, two chromatin remodeling proteins and a histone deacetylase, as collaborators of condensin for chromosome condensation (Robellet et al., 2014). In line with that, we identified chromatin remodeling enzymes (CHR17, CRH19 and BRAHMA), histone chaperones (NAP1;1 and NAP1;2), a histone deacetylase (HDC1) and a histone acetyltransferase (ELO3) in the affinity purification experiments with CAP-D2 and CAP-D3. All of them are chromatin modifiers important for plant development (Perrella et al., 2013; Skylar et al., 2013; Gentry and Hennig, 2014).

\section{Condensin I is located within the cytoplasm and nuclei during interphase}

During interphase, in vertebrates the most commonly described localization of condensin I is exclusively in the cytoplasm (Hirota et al., 2004; Ono et al., 2004; Gerlich et al., 2006; Hirano, 
2012a). However, some reports regarding Drosophila, chicken and human cell cultures described the localization of condensin I additionally within the nucleus (Schmiesing et al., 2000; Savvidou et al., 2005; Zhang et al., 2016). In A. thaliana protoplasts and $N$. benthamiana epidermal leaves, we observed CAP-D2, CAP-G and CAP-H EYFP-fusion proteins in the cytoplasm as well as the nucleus. The cytoplasmic localization of CAP-H was already described (Fujimoto et al., 2005), but not yet its nuclear localization.

In stable A. thaliana transformants carrying CAP-D2 or CAP-D3 tagged at its $\mathrm{N}$ - or C-terminus to EYFP and in stable cap- $d 3$ mutants carrying CAP-D3-EYFP, the fusion proteins could not be visualized, neither directly nor indirectly. The constructs are functionally active, since they work in $A$. thaliana and in $N$. benthamiana after transient transformations. In addition, the CAP-D3-EYFP construct was able to complement the centromeric phenotype of the cap-d3SALK mutants. Similar problems have been described for GFP-PATRONUS1 A. thaliana transformants (Zamariola et al., 2014). These authors suggested that the reason behind could be the low expression or stability of the PATRONUS protein due to the presence of an APC/C degradation box. However, in CAP-D2 no APC/C degradation box exists. The detection of CAP-D2 in leaves from A. thaliana wild-type plants by Western blot was also not possible. This may be due to a low protein level in wild-type leaves since the transcript level in leaves is very low. By Western blot the CAP-D2 protein was detectable in protoplasts only when constitutively overexpressed. Similarly, in Drosophila the detection of condensin from extracts of non-dividing tissues was also not possible (Cobbe et al., 2006).

\section{CAP-D3 may influence interphase chromatin arrangement and transcription, but not histone modifications}

In Drosophila, CAP-D3 and CAP-H2 are needed to form compact chromosomes (Hartl et al., 2008; Bauer et al., 2012). Condensins via maintaining chromatin condensation may also maintain nuclear shape and size, as indicated after SMC2, CAP-H2 and CAP-D3 depletion in human cells (George et al., 2014). In embryonic stem cells of mice, the depletion of SMC2 causes chromatin decondensation as well as the increase of the nuclear volume (Fazzio and Panning, 2010). On the other hand, in C. elegans, the depletion of SMC4, CAP-G2 or HCP-6 (CAP-D3) does not change the chromosome territory volumes (Lau et al., 2014).

In A. thaliana, previous studies based on FISH suggested an influence of CAP-D3 on the formation of the top arm 1 interphase chromosome territories and sister chromatid cohesion (Schubert et al., 2013). Using FISH probes against a smaller part of chromosome 1 bottom arm, we could not detect an increase of the hybridization signal area in the cap- $d 3$ mutants compared to wild-type plants. The differences could be explained by labeling only one fourth of the chromosome arm by FISH, while in the previous study the whole chromosome arm (without pericentromeric heterochromatin) was visualized. The different methods used to quantify the dispersion of the interphase chromatin could be another reason. The degree of chromatin condensation within nuclei may depend on the type of tissue (Tessadori et al., 2007; van Zanten et al., 2011). Light (Bourbousse et al., 2015), drought, temperature, and salinity stress, as well as toxic components, energy-rich radiation and chemically induced DNA damage may also induce dynamic structural changes in plant chromatin (reviewed in Probst \& Mittelsten-Scheid, 2015). Even compressive stress has the potential to reorganize chromatin (Damodaran et al., 2018; Xia et al., 2018). Thus, these factors have also the potential to influence chromatin condensation in the CAP-D mutants.

Although we could not confirm the euchromatin dispersion in the cap-d3 mutants, it cannot be excluded that CAP-D3 is involved in the organization of chromosome territories as found in Drosophila (Bauer et al., 2012; Hirano, 2012b). The mutants used in our analysis (cap-d3 SAIL and cap-d3 $S A L K$ ) have knockdown alleles, meaning that there is still a truncated transcript that could produce a partially functional CAP-D3 protein (Schubert et al., 2013).

In addition to its role in chromosome compaction, condensin II has been described to influence transcription (Longworth et al., 2012; Dowen et al., 2013; Yuen et al., 2017). Although the A. thaliana cap- $d 3$ mutants showed only moderate transcriptional changes, CAP-D3 might still affect the expression of genes involved in transcription and response to stress. This conclusion arises from our observation on the cap-d3 mutants which die sooner than wild-type plants after stress, such as pathogen infection. Interestingly, gross chromosome rearrangements altering the genome topology do not alter gene expression in Drosophila (Ghavi-Helm et al., 2018). Even a budding yeast strain, after merging all 16 chromosomes into a single one, revealed a nearly identical transcriptome and similar 
phenome profiles as wild-type strains (Shao et al., 2018). Thus, chromatin structure changes as induced in the $A$. thaliana cap- $d 3$ mutants seem to influence the global transcription only slightly.

Wang et al. (2017) showed that A. thaliana SMC4, but not CAP-D3, is important to maintain the repression of pericentromeric retrotransposons independent of DNA methylation. Accordingly, we observed no increased retrotransposon transcription in any of the cap- $d 3$ mutants. Moreover, in accordance with our observations for both cap- $d 3$ mutants, the protein-coding genes up-regulated in $s m c 4$ mutants are mainly involved in flower development, reproductive processes and DNA repair, and are distributed all over the genome (Wang et al., 2017). However, we observed in the cap-d3 mutants a differential expression of genes involved in transcription and stress response. This difference could be due to the combined effects of both condensin complexes I and II in the smc4 mutants, while in our cap- $d 3$ mutants only condensin II is compromised.

Posttranslational histone modifications may affect the structure and stiffness of interphase nuclei, and decondensed euchromatin correlates with less rigid nuclei (Chalut et al., 2012; Krause et al., 2013; Haase et al., 2016). In human HeLa cells histone methylation, but not acetylation, contributes to the stiffness and structure of condensed mitotic chromosomes (Biggs et al., 2019). Histone acetylation relaxes chromatin allowing different protein complexes to access DNA. Thus, acetylation is associated with transcription, and hypoacetylation with transcriptional repression (Wang et al., 2014).

It seems that the unaltered degree and pattern of histone acetylation reflects an only moderate effect on transcription as we observed in the capd-3 mutants.

\section{CAP-D proteins prevent heterochromatin clustering}

CAP-H2 promotes the spatial separation of heterochromatic regions in Drosophila during interphase (Bauer et al., 2012; Buster et al., 2013). Correspondingly, in A. thaliana, depletion of CAP-D3 results in centromere clustering at interphase (Schubert et al., 2013). We confirmed this interphase phenotype and found that CAP-D3 depletion also results in the clustering of the 45S rDNA loci but not of the 5S rDNA sites. A differential behavior of rDNA was also found in protoplasts of $A$. thaliana. 45S rDNA remains condensed while the 5S rDNA decondenses during protoplast formation (Tessadori et al., 2007). 5S and 45S rDNA are transcribed by RNA polymerases III and I, respectively (Layat et al., 2012). Therefore, the different clustering behaviors of both rDNAs in the cap-d3 mutants could be due to their different structural and functional properties. Moreover, condensin of fission yeast, which is similar to condensin I, binds to RNA polymerase III transcribed genes (5S rDNA and tRNA), and mediates their localization near the centromeres (Iwasaki et al., 2010).

The nuclear and chromocenter phenotype which we observed in the cap-d3 mutants differs from previous reports (Moissiard et al., 2012; Sakamoto and Takagi, 2013; Tatout et al., 2014; Poulet et al., 2017; Wang et al., 2017). The chromocenters cluster and localize at the nuclear periphery, but do not decondense, the nuclear area does not change compared to that of wild-type, and the general degree of DNA and histone methylation is unaffected. Moreover, hypomethylation, linc and morc mutants do not show transcriptional silencing of centromeric and pericentromeric repeats, and of silenced genes (Moissiard et al., 2012; Poulet et al., 2017). In contrast, CAP-D3 has little effect on silencing, because no increased transcription of transposable elements was detected in cap-d3 mutants (Wang et al., 2017). MORC, CRWN and LINC proteins localize close to the chromocenters, MORC foci adjacent to the chromocenters (Moissiard et al., 2012), CRWN1 and CRWN4 at the nuclear periphery (Sakamoto and Takagi, 2013), and the LINC complex in the nuclear envelope (Tatout et al., 2014). Conversely, CAP-D3 influences the arrangement of the chromocenters but localizes exclusively in euchromatin during interphase (Schubert et al., 2013). Therefore, CAP-D3 has mainly a structural role during interphase and affects the clustering of chromocenters without localizing close to them.

Statistical analysis detected a more regular, than a completely random spatial centromere and chromocenter distributions in animal and plant nuclei. This suggests that repulsive constraints or spatial inhomogeneities influence the 3D organization of heterochromatin (Andrey et al., 2010). Computer simulation modeling of A. thaliana chromosomes as polymers predicts that the position of the chromocenters in the nucleus is due to non-specific interactions (de Nooijer et al., 2009). The simulated chromosomes exhibit chromocenter clustering except for the so-called Rosette chromosomes, in which the euchromatin loops emanate from the chromocenter and thus prevent chromocenter clustering (Fransz et al., 2002). Indeed, depletion-attraction forces predict that big particles in an environment crowded with small particles will tend to cluster together (Marenduzzo et 
al., 2006). This situation can be applied to the nucleus where the chromocenters act as big particles and euchromatin as small particles. If association is not prevented, the chromocenters will cluster.

In cap- $d 3$ mutants we observed chromocenter clustering but barely chromosome territory dispersion. During mitosis, CAP-D3 is needed to confer the rigidity of the chromosome arms (Green et al., 2012) and human condensin controls the elasticity of mitotic chromosomes (Sun et al., 2018).

We suppose, that during interphase, CAP-D3 localizes in euchromatin, possibly along the euchromatic loops, mediating the rigidity which is needed to keep the chromocenters away from each other. In case of lacking or functionally impaired CAP-D3, the loops may be not stiff enough to prevent the chromocenter clustering while the chromosome territories may mainly keep their structures (Fig. 9). The finding that condensed chromatin resist to mechanical forces, whereas decondensed chromatin is more soft (Maeshima et al., 2018) supports the idea that the stiffness of chromatin is an important feature to organize cell nuclei. Our observation that the degree of methylation and acetylation is not altered in the cap- $d 3$ mutants suggests that these post-translational histone modifications are not required for the rigidity of interphase chromosome territory structures.

\section{EXPERIMENTAL PROCEDURES}

\section{Plant material and stable transformation}

All Arabidopsis thaliana (L.) Heynh lines and control plants are in Columbia-0 (Col-0) background. The T-DNA cap-d3 (SAIL_826_B06, SALK_094776) insertion lines were previously described and selected in our laboratory (Schubert et al., 2013). Seeds were sown in soil and germinated under shortday conditions $\left(16 \mathrm{~h}\right.$ dark $/ 8 \mathrm{~h}$ light, $\left.18-20^{\circ} \mathrm{C}\right)$ and then transferred to long-day conditions $(16 \mathrm{~h} \mathrm{light} / 8$ $\mathrm{h}$ dark, $18-20^{\circ} \mathrm{C}$ ) before bolting. The lines were genotyped by PCR using the primers listed in Table $\mathrm{S} 4$. The presence of the T-DNA was further confirmed by sequencing.

A. thaliana stable transformants were generated by the floral dip method (Clough and Bent, 1998). For selection of primary transformants, the seeds were sterilized and plated on $1 / 2$ Murashige and Skoog (MS) basal medium (Sigma) supplemented with the adequate antibiotics when required and grown in a growth chamber under-long day conditions.

\section{Transcript quantification}

For transcript quantification total RNA was extracted from leaves, roots, 7 days-old seedlings and flower buds with the RNeasy Plant Mini kit (Qiagen) following manufacturer's instructions. All RNA samples were treated with TURBO DNAse (Thermo Fisher Scientific) and tested for DNA contamination by PCR. Reverse transcription was performed using 250 ng of total RNA and the RevertAid H Minus First Strand cDNA Synthesis kit (Thermo Fischer Scientific), with oligo(dT)18 primers, according to manufacturer's instructions. The quality of the cDNA was checked with a PCR targeting EF1B mRNA (Elongation factor $1 \beta$ ).

Quantitative RT-PCRs for $C A P-D 2$ and $C A P-D 3$ transcripts were done in triplicates and from three independent biological samples using SYBR ${ }^{\mathrm{TM}}$ Green PCR Master Mix (Thermo Fischer Scientific) in a 7900HT Fast Real-Time PCR System (Applied Biosystems). For each reaction, $0.5 \mu 1$ of cDNA template and $0.6 \mathrm{mM}$ primers (Table S4) were used in $10 \mu 1$. PPA2 and At4g26410 (Kudo et al., 2016) were used as reference genes for data normalization and the data were analyzed with the Double Delta Ct method (Livak and Schmittgen, 2001).

\section{$C A P-D 2$ and $C A P$-D3 promoter::GUS reporter lines and $\beta$-glucuronidase activity assay}

Different lengths of the promoter regions of both $C A P-D 2$ and $C A P-D 3$ were cloned between the SalI and NotI restriction sites of the pEntr 1A plasmid (Invitrogen). The sequences were amplified from gDNA with the primer pairs D2-1156F/D2ProR for the Pro4 fragment, D2-1156F/D2Int1R for Pro5, D2-1156F/D2Int2R for Pro6, D2-392F/D2ProR for Pro7, D2-392F/D2Int1R for Pro8, D2392F/D2Int2R, for Pro9, D3-1318F/D3ProR for Pro10 and D3-474F/D3ProR for Pro11 (Table S4). The fragments were subcloned upstream of the GUS reporter gene in the pGWB633 plasmid (Nakamura et al., 2010) using the Gateway LR Clonase II enzyme mix (Invitrogen) following manufacturer instructions.

Constructs were transformed into A. thaliana and stable transformants were selected in $1 / 2$ MS (Sigma) with $16 \mathrm{mg} / \mathrm{L}$ PPT (Duchefa). One month after sowing, the plantlets were stained for GUS activity according to Jefferson et al. (1987) with small modifications. Plantlets were collected in $15 \mathrm{ml}$ tubes 
containing $1 \% \quad \mathrm{X}$-Glu (5-Bromo-4-Chloro-3-indolyl- $\beta$-D-Glucopyranoside; Duchefa) in $0.1 \mathrm{M}$ phosphate buffer ( $\mathrm{pH}$ 7.0). To facilitate the penetration of the solution in the material, the tubes with the plant material and the staining solution were exposed to vacuum for $5 \mathrm{~min}$ and incubated overnight at $37^{\circ} \mathrm{C}$. Next day, the staining solution was replaced by $70 \%$ ethanol and incubated $20 \mathrm{~min}$ at $60^{\circ} \mathrm{C}$. This step was repeated until the chlorophyll was removed. The stained material was preserved in $70 \%$ ethanol at $4 \mathrm{C}^{\circ}$ and analyzed under a stereo microscope.

\section{Condensin subunit EYFP-fusion constructs}

The 3942 bp and 4245 bp long cDNA sequences of CAP-D3 and CAP-D2 respectively, were synthesized and cloned into pEntr 1A (Invitrogen) by a DNA-Cloning-Service (Hamburg, Germany). The $3153 \mathrm{bp}$ and the $2013 \mathrm{bp}$ long cDNA sequences of $C A P-H$ and $C A P-G$ were amplified from flower bud cDNA with the primer pairs CAPH_pentry_f/CAPH_pentry_r and CAPG_pEnt_f/CAPG_pEntr_r (Table S4), respectively, and cloned between the SalI and Notl sites of the pEntr 1A plasmid (Invitrogen).

Once in the pEntr 1A plasmid, the coding sequences of the genes of interest were subcloned into pGWB641 and pGWB642 plasmids (Nakamura et al., 2010) using Gateway cloning (Invitrogen). The generated expression cassettes contained the proteins of interest fused to EYFP C-terminally for the pGWB641 constructs (CAP-D2_EYFPc, CAP-D3_EYFPc, CAP-G_EYFPc and CAP-H_EYFPc) or N-terminally for the pGWB642 constructs (CAP-D2_EYFPn and CAP-D3_EYFPn), and both were under the control of the cauliflower mosaic virus 35S promoter. As a control (Control_EYFPc), a plasmid containing only EYFP under the control of the $35 \mathrm{~S}$ promoter was generated.

\section{Condensin I subunit localization in A. thaliana protoplasts and $N$. benthamiana}

Isolation and transformation of A. thaliana leaf protoplasts were performed as described in Yoo et al., (2007). To improve the visualization of the fusion proteins, the transformed protoplasts were fixed in $4 \%$ formaldehyde in $1 \times \mathrm{PBS}$, washed in $1 \times \mathrm{PBS}$ and centrifuged at $400 \mathrm{rpm}$ for $5 \mathrm{~min}$ (Shandon CytoSpin3, GMI inc) onto a microscopic slide. The slide was directly used for immunostaining against EYFP.

Nicotiana benthamiana leaf cells were transformed as described in Sparkes et al., (2006). Agrobacteria carrying the constructs of interest were grown in YEB medium with suitable antibiotics to an $\mathrm{OD}_{600}$ of 1 and resuspended in infiltration medium (10 mM MES, $10 \mathrm{mM} \mathrm{MgCl}$, pH 5.6, 3.3 $\mathrm{mM}$ acetosyringone). $N$. benthamiana leaves of 2 to 3 weeks old plants were infiltrated with the Agrobacterium suspension using a syringe without needle and analyzed 2 to 4 days later.

\section{Antibody production}

The sequence between 2743 and 4248 bp of CAP-D2 (501 C-terminal amino acids) was amplified from A. thaliana flower bud cDNA with the D2CtSalI_F and D2CtNotlI_R primers (Table S4). The fragment was cloned between the SalI and NotI restriction sites of the pET23a(+) plasmid (Novagen) resulting in a pEt23_CAP-D2_Ct construct which contains the cassette T7 promoter::T7 tag-CAP$D 2 \mathrm{Ct}$-His tag. The construct was transformed into E. coli BL21 cells and the expression of the transgene induced with $1 \mathrm{mM}$ IPTG (isopropyl- $\beta$-D-thiogalatopyranoside, Sigma-Aldrich). The recombinant protein was purified with agarose beads that bind specifically to the His-tag (Ni-NTA Agarose, Qiagen) following the purification hybrid method from the ProBond purification system (Thermo Fisher Scientific). The purified recombinant protein was used to produce anti-CAP-D2 polyclonal antibodies in rabbit (Udo Conrad, Phytoantibody group, IPK, Gatersleben, Germany). Two rabbits were immunized with the recombinant proteins and the anti-serum collected after four immunizations. The anti-CAP-D2 serum was used directly for Western blot.

\section{Total protein extraction and Western blot}

Isolated protoplast or grinded plant leaf material were resuspended in 100-300 $\mu 1$ of protein extraction buffer (56 mM Na $\mathrm{CO}_{3}, 56 \mathrm{mM}$ DTT, 2\% SDS, 12\% Sucrose, 2 mM EDTA, bromophenol blue), incubated $20 \mathrm{~min}$ at $65^{\circ} \mathrm{C}$ and centrifuged at high speed $(13,000 \mathrm{rpm})$. Then, the supernatant containing the soluble total protein was used for Western blot analysis.

For Western blot, the extracted proteins were separated in 10\% polyacrylamide gels (Schägger and von Jagow, 1987) and blotted onto Immobilion-Fl membranes (Millipore). The membranes were incubated in blocking solution (5\% skim milk in TBST) for $30 \mathrm{~min}$ to reduce non-specific binding of 
the antibodies. Then, the membranes were incubated overnight at $4{ }^{\circ} \mathrm{C}$ with the adequate primary antibodies in TBST buffer: mouse anti-His-tag (1:2000, Millipore, 05-949), rabbit anti-CAP-D2 serum (1:1000) or rabbit anti-GFP conjugated with Alexa488 (1:1000, Chromotek, PABG1). And then with secondary antibodies: anti-mouse IgG IRDye 680RD (1:10000, LI-COR, 926-32222) or anti-rabbit IgG IRDye800CW (1:5000, LI-COR, 925-32213). The membranes were imaged using a LI-COR Odyssey Imager (LI-COR). For T7-tag detection, the membranes were first incubated with anti-T7-tag conjugated with alkaline phosphatase $(1: 10000$, Merck, \#6999) for $1 \mathrm{~h}$, and then with phosphateactivity buffer $(100 \mathrm{mM}$ Tris, $100 \mathrm{mM} \mathrm{NaCl}, 1 \mathrm{mM} \mathrm{MgCl} 2,0.33 \mathrm{mg} / \mathrm{ml}$ nitro blue tetrazolium and 0.17 $\mathrm{mg} / \mathrm{ml}$ 5-bromo-4-chloro-3-indolyl phosphate) in the dark until the signals were visible.

\section{Affinity purification and analysis of GS-tagged CAP-D2 and CAP-D3 from PSB-D cells}

The cDNA sequences of $C A P-D 3$ and $C A P-D 2$ were synthesized and cloned into pCambia 2300 35S GS-Ct by the DNA-Cloning-Service (Hamburg, Germany) resulting in the constructs pCambia2300_CAP-D2_GS and pCambia2300_CAP-D3_GS.

The A. thaliana ecotype 'Landsberg erecta' cell suspension (PSB-D) was transformed as described (Van Leene et al., 2011). CAP-D2-GS and CAP-D3-GS were affinity purified following the protocol described (Dürr et al., (2014).

For mass spectrometry, the eluted proteins were separated in a $10 \%$ polyacrylamide gel and digested with trypsin. Mass spectrometry and data analysis were performed according to Antosz et al., (2017). Protein Scape 3.1.3 (Bruker Daltonics) in connection with Mascot 2.5.1 (Matrix Science) facilitated database searching of the NCBInr database.

Three independent affinity purifications were performed. A MASCOT score of minimum 100 and the presence in at least two of the purifications were considered as criteria for reliable protein identification. The experimental background (contaminating proteins that co-purified with the unfused GS-tag) and non-specific interactions (proteins that co-purified independently of the bait used) were subtracted. The list of non-specific A. thaliana proteins is based on 543 affinity purification experiments using 115 different baits (Van Leene et al., 2014).

\section{Nuclei preparations}

A. thaliana nuclei from differentiated leaf cells were isolated and flow-sorted according to their ploidy level as described (Weisshart et al., 2016) in a BD INFLUX Cell Sorter (BD Bioscience). The nuclei were sorted based on their DNA content in $2 \mathrm{C}, 4 \mathrm{C}, 8 \mathrm{C}$ and $16 \mathrm{C}$ ploidy fractions. Twelve $\mu \mathrm{l}$ of $4 \mathrm{C}$ sorted nuclei and the same amount of sucrose buffer $\left(10 \mathrm{mM}\right.$ Tris, $50 \mathrm{mM} \mathrm{KCl}, 2 \mathrm{mM} \mathrm{MgCl}-6 \mathrm{H}_{2} \mathrm{O}$, $5 \%$ sucrose, $\mathrm{pH} 8.0$ ) were placed on a slide. The slides were directly used or stored at $-20^{\circ} \mathrm{C}$.

A. thaliana nuclei were embedded in acrylamide to preserve their $3 \mathrm{D}$ structure following the procedure described by Kikuchi et al., (2005) with modifications. Twelve $\mu 1$ of nuclei suspension were mixed on a slide with $6 \mu$ l of active $15 \%$ acrylamide embedding medium (15\% acrylamide/bisacrylamide (29:1), $15 \mathrm{mM}$ PIPES, $80 \mathrm{mM} \mathrm{KCl}, 20 \mathrm{mM} \mathrm{NaCl}, 2 \mathrm{mM}$ EDTA, $0.5 \mathrm{mM}$ EGTA, $0.5 \mathrm{mM}$ spermidine, $0.2 \mathrm{mM}$ spermine, $1 \mathrm{mM}$ DTT, $0.32 \mathrm{M}$ sorbitol, $2 \%$ APS and $2 \% \mathrm{Na}_{2} \mathrm{SO}_{3}$ ). A coverslip was carefully placed on top of the acrylamide-nuclei mixture and let to polymerize $30 \mathrm{~min}$ at room temperature. The coverslip was then removed letting a thin pad of nuclei embedded in acrylamide on the slide that was directly used for FISH.

\section{Preparation of squashed $A$. thaliana roots}

A. thaliana seedlings were fixed in $4 \%$ paraformaldehyde in phosphate-buffered saline buffer $(1 \times \mathrm{PBS}$ buffer). The seedlings were washed in $1 \times \mathrm{PBS}$ buffer and digested for $30 \mathrm{~min}$ at $37^{\circ} \mathrm{C}$ in an enzyme mix $(0.5 \%$ pectolyase (Sigma), $0.5 \%$ cytohelicase (Sigma), $0.35 \%$ cellulase (Calbiochem), $0.35 \%$ cellulase (Duchefa) in $1 \times$ PBS buffer. After removal of the enzyme solution and washing in $1 \times$ PBS, the root tips were transferred to a slide and squashed between coverslip and slide. The liquid nitrogen frozen coverslip was lifted and the slide directly used for immunostaining.

\section{Probe preparation and fluorescence in situ hybridization (FISH)}

The probes were generated by: (i) PCR for the 180 bp centromeric repeat (pAL; Martinez-Zapater et al., 1986), (ii) from a plasmid for the 5S rDNA probe (pCT4.2; Campell et al., 1992), (iii) from BACs containing the 45S rDNA repeats (BAC T15P10), and (iv) for painting a part of chromosome territory 1 bottom (CT1B) from BACs arranged in contigs (BACs F11P17 to F12B7). The BACs were obtained from the Arabidopsis Biological Resource Center (Ohio, USA). The probes were labeled with 
modified dUTPs conjugated with Texas-red (Invitrogen) or Alexa-488 (Invitrogen) by nicktranslation. The FISH was performed as previously described (Pecinka et al., 2004).

\section{Indirect immunofluorescence labeling}

Nuclei and chromosome preparations were washed in $1 \times \mathrm{PBS}$ and incubated for $30 \mathrm{~min}$ at $37^{\circ} \mathrm{C}$ in a moist chamber with $30 \mu$ l blocking buffer (4\% BSA, $0.1 \%$ Tween-20 in $1 \times \mathrm{PBS}$ ) to reduce nonspecific antibody binding. After three washes in $1 \times \mathrm{PBS}$, the slides were incubated with the primary antibodies diluted in antibody buffer (1\% BSA, $0.1 \%$ Tween-20 in $1 \times \mathrm{PBS})$ overnight at $4^{\circ} \mathrm{C}$. Next day, the slides were washed in $1 \times \mathrm{PBS}$ again and incubated with the secondary antibodies in antibody buffer for $1 \mathrm{~h}$ at $37^{\circ} \mathrm{C}$. After incubation, the preparations were washed in $1 \times \mathrm{PBS}$, dehydrated in an ethanol series $(70 \%, 90 \%$ and $96 \%$ ethanol for 2 min each) and counterstained with DAPI in antifade (Vectashield). All primary and secondary antibodies, and the dilutions used are listed in Table S5.

Immunolocalization of 5-methyl-cytosine requires an initial DNA denaturation of the specimen. Therefore, slides with sorted nuclei were denatured in $70 \%$ formamid in $2 \times \mathrm{SSC}$ for 2 min at $70^{\circ} \mathrm{C}$. The preparations were dehydrated in ice cold $70 \%$ and $96 \%$ ethanol for $5 \mathrm{~min}$ each, and air-dried. Subsequent blocking and antibody incubation were carried as described above.

\section{Microscopy and image analysis}

Wide-field fluorescence microscopy was used to evaluate and image the nuclei and chromosome preparations with an Olympus BX61 microscope (Olympus) and an ORCA-ER CCD camera (Hamamatsu). When higher resolution was needed to analyze the spatial arrangement of the chromocenters, a super-resolution fluorescence microscope Elyra PS.1 and the software ZEN (Carl Zeiss $\mathrm{GmbH}$ ) was used. Processing and analysis of microscopic image stacks were done with ZEN, Adobe Photoshop CS5 (Adobe) and Imaris 8.0 (Bitplane) software.

The CT1B signals were quantified on 16-bit gray scale microscopic images using ImageJ v1.50i (Schneider et al., 2012). The images were taken from preparations of flow-sorted nuclei. Since this technique flattens the nuclei, they were considered as two-dimensional. Within each dataset all images were treated the same way after using the same acquisition parameters. For the CT1B signal image dataset, the background was subtracted with the option 'Rolling ball' set at 25 pixels and the delimitation of the region of interest (ROI) with the RenyiEntropy threshold. For the nuclear area image dataset (measured based on DAPI staining), the background was not subtracted, and the nuclear area was delimited as a ROI also with the RenyiEntropy threshold. The area of each ROI was automatically measured by the program.

\section{Southern blot analysis}

Five $\mu \mathrm{g}$ of genomic DNA from A. thaliana leaves was digested with either HpaII or MspI (Thermo Fischer Scientific). The DNA was gel-separated and transferred onto a nylon membrane (Hybond XL, Amersham). The ${ }^{32} \mathrm{P}$-labelled centromeric 180 bp repeat pAL was used for Southern hybridization and the signals were detected by autoradiography. The A. thaliana centromeric pAL probe was generated by PCR and ${ }^{32} \mathrm{P}$-labeled according to manufacturer's instructions (Deca-Label DNA labelling Kit, Thermo Scientific).

\section{cap-d3 RNA-seq and in silico transcriptome analysis}

cap-d3 SAIL, cap-d3SALK and control (Col-0) seeds were sown in soil and grown under short day conditions. RNA was extracted with the RNeasy Plant Mini Kit (Qiagen) from $50 \mathrm{mg}$ of pooled 4 weeks old plantlets cut above the root. For each of the three A. thaliana genotypes five independent RNA extractions were performed and the RNA integrity of the samples was measured in a 2100 Bioanalyzer (Agilent). The four RNA samples of each genotype with the highest RIN (RNA Integrity Number) were used for library preparation and RNA sequencing (NGS platform, IPK Gatersleben, Germany). The libraries were prepared with a TruSeq RNA Library Kit (Illumina) unstranded and sequenced in a HiSeq2000 system (single 100 bp reads).

The quality of the RNA-seq reads were assessed with FastQC v0.11.4 (Babraham Bioinformatics) and adaptors trimmed with Trimmomatic v0.32 (Bolger et al., 2014). After a second quality check in FastQC, the reads were aligned with GSNAP v2016-05-25 (Wu and Nacu, 2010) against the Arabidopsis TAIR10 genome and the gene counts calculated with HTseq v0.6.1 (Anders et al., 2015). Differential expression analyses were performed using the DESeq2 1.14.0 Bioconductor package (Love et al., 2014). Genes were considered differentially expressed (DEG) when they had a 
Benjamini-Hochberg-adjusted- $P$ value $\leq 0.05$ and a $\log _{2}$-fold change $\leq-1$ or $\geq 1$. These steps were performed through Galaxy (Afgan et al., 2018).Genes detected as differentially expressed for both cap-d3 mutants were considered as the genes associated to CAP-D3 defective proteins independently of the specific mutation. Gene enrichment was analyzed with agriGO v1.2 (Du et al., 2010). The analysis of the transcription factors present in cap- $d 3$ DEG was perform with the Arabidopsis Transcription Factor Database (AtTFDB) from the Arabidopsis Gene Regulatory Information Server (AGRIS; Yilmaz et al., 2011).

Gene and protein identification numbers

Sequence data from this study can be found in The Arabidopsis Information Resource (TAIR, www.arabidopsis.org) or National Center for Biotechnology Information (NCBI, www.ncbi.nlm.nih.gov/) databases under the following gene identification numbers: CAP-D2, AT3G57060; CAP-D3, AT4G15890; CAP-G, AT5G37630; CAP-H, AT2G32590.

\section{ACKNOWLEDGEMENTS}

We thank Jörg Fuchs for flow sorting of nuclei, Katrin Kumke, Oda Weiss, Karla Meier and Ulrike Gresch for excellent technical assistance, Axel Himmelbach for sequencing, and Ingo Schubert for critical reading of the manuscript. The work was supported by a Marie Curie Initial Training Network fellowship (FP7-PEOPLE-2013-ITN, CHIP-ET).

\section{Author contributions}

VS, CM, AH and KG conceived the study and designed the experiments. AH and VS contributed equally to supervise the project. CM, WA, and VS performed the experiments. CM, EK, MVB, IE, $\mathrm{FC}, \mathrm{AB}$ and IL performed the bioinformatic analysis. CM and VS wrote the manuscript. All authors read and approved the final manuscript.

\section{REFERENCES}

Afgan, E., Baker, D., Batut, B., Van Den Beek, M., Bouvier, D., Čech, M., Chilton, J., Clements, D., Coraor, N., Grüning, B.A. and Guerler, A. (2018) The Galaxy platform for accessible, reproducible and collaborative biomedical analyses: 2018 update. Nucleic Acids Res., 46, W537-W544.

Anders, S., Pyl, P.T. and Huber, W. (2015) HTSeq-a Python frame work to work with highthroughtput sequencing data. Bioinformatics, 31, 166-169.

Andrey, P., Kieu, K., Kress, C., Lehmann, G., Tirichine, L., Liu, Z.C., Biot, E., Adenot, P.G., Hue-Beauvais, C., Houba-Herin, N., Duranthon, V., Devinoy, E., Beaujean, N., Gaudin, V., Maurin, Y. and Debey, P. (2010) Statistical analysis of 3D images detects regular spatial distributions of centromeres and chromocenters in animal and plant nuclei. PloS Computat. Biol., 6, e1000853.

Antosz, W., Pfab, A., Ehrnsberger, H.F., Holzinger, P., Kollen, K., Mortensen, S.A., Bruckmann, A., Schubert, T., Langst, G., Griesenbeck, J., Schubert, V., Grasser, M. and Grasser, K.D. (2017) The composition of the Arabidopsis RNA polymerase II transcript elongation complex reveals the interplay between elongation and mRNA processing factors. Plant Cell, 29, 854-870.

Bauer, C.R., Hartl, T.A. and Bosco, G. (2012) Condensin II promotes the formation of chromosome territories by inducing axial compaction of polyploid interphase chromosomes. PLoS Genet., 8, e1002873.

Berr, A. and Schubert, I. (2007) Interphase chromosome arrangement in Arabidopsis thaliana is similar in differentiated and meristematic tissues and shows a transient mirror symmetry after nuclear division. Genetics, 176, 853-863.

Biggs, R., Liu, P.Z., Stephens, A.D. and Marko, J.F. (2019) Effects of altering histone posttranslational modifications on mitotic chromosome structure and mechanics. Mol. Biol. Cell, 30, 820-827. 
Bolger, A.M., Lohse, M. and Usadel, B. (2014) Trimmomatic: a flexible trimmer for Illumina sequence data. Bioinformatics, 30, 2114-2120.

Bourbousse, C., Mestiri, I., Zabulon, G., Bourge, M., Formiggini, F., Koini, M.A., Brown, S.C., Fransz, P., Bowler, C. and Barneche, F. (2015) Light signaling controls nuclear architecture reorganization during seedling establishment. Proc. Natl. Acad. Sci. $U S$ A, 112, E2836-2844.

Boyle, S., Gilchrist, S., Bridger, J.M., Mahy, N.L., Ellis, J.A. and Bickmore, W.A. (2001) The spatial organization of human chromosomes within the nuclei of normal and emerinmutant cells. Hum. Mol. Genet., 10, 211-219.

Buster, D.W., Daniel, S.G., Nguyen, H.Q., Windler, S.L., Skwarek, L.C., Peterson, M., Roberts, M., Meserve, J.H., Hartl, T., Klebba, J.E., Bilder, D., Bosco, G. and Rogers, G.C. (2013) SCFSlimbubiquitin ligase suppresses condensin II-mediated nuclear reorganization by degrading Cap-H2. J. Cell Biol., 201, 49-63.

Campell, B.R., Song, Y., Posch, T.E., Cullis, C.A. and Town, C.D. (1992) Sequence and organization of $5 \mathrm{~S}$ ribosomal RNA-encoding genes of Arabidopsis thaliana. Gene, 112, $225-228$.

Chalut, K.J., Hopfler, M., Lautenschlager, F., Boyde, L., Chan, C.J., Ekpenyong A., Martinez-Arias, A. and Guck, J. (2012) Chromatin decondensation and nuclear softening accompany Nanog downregulation in embryonic stem cells. Biophys. J., 103, 2060-2070.

Clough, S.J. and Bent, A.F. (1998) Floral dip: A simplified method for Agrobacteriummediated transformation of Arabidopsis thaliana. Plant J., 16, 735-743.

Cobbe, N. and Heck, M.M.S. (2004) The evolution of SMC proteins: phylogenetic analysis and structural implications. Mol. Biol. Evol., 21, 332-347.

Cobbe, N., Savvidou, E. and Heck, M.M.S. (2006) Diverse mitotic and interphase functions of condensins in Drosophila. Genetics, 172, 991-1008.

Cremer, T. and Cremer, M. (2010) Chromosome territories. Cold Spring Harbor Perspectives in Biology, 2, a003889.

Cremer, T., Cremer, M. and Cremer, C. (2018) The 4D nucleome: genome compartmentalization in an evolutionary context. Biochemistry (Mosc), 83, 313-325.

Damodaran, K., Venkatachalapathy, S., Alisafaei, F., Radhakrishnan, A.V., Sharma Jokhun, D., Shenoy, V.B. and Shivashankar, G.V. (2018) Compressive force induces reversible chromatin condensation and cell geometry dependent transcriptional response. Mol. Biol. Cell, 29, 3039-3051.

De Nooijer, S., Wellink, J., Mulder, B. and Bisseling, T. (2009) Non-specific interactions are sufficient to explain the position of heterochromatic chromocenters and nucleoli in interphase nuclei. Nucleic Acids Res., 37, 3558-3568.

Doğan, E.S. and Liu, C. (2018) Three-dimensional chromatin packing and positioning of plant genomes. Nat. Plants, 8,521-529.

Dowen, J.M., Bilodeau, S., Orlando, D.A., Hübner, M.R., Abraham, B.J., Spector, D.L. and Young, R.A. (2013) Multiple structural maintenance of chromosome complexes at transcriptional regulatory elements. Stem Cell Reports, 1, 371-378.

Du, Z., Zhou, X., Ling, Y., Zhang, Z. and Su, Z. (2010) agriGO: a GO analysis toolkit for the agricultural community. Nucleic Acids Res., 38, W64-70.

Dürr, J., Lolas, I.B., Sørensen, B.B., Schubert, V., Houben, A., Melzer, M., Deutzmann, R., Grasser, M. and Grasser, K.D. (2014) The transcript elongation factor SPT4/SPT5 is involved in auxin-related gene expression in Arabidopsis. Nucleic Acids Res., 42, 4332-4347.

Elbatsh, A.M.O., Kim, E., Eeftens, J.M., Raaijmakers, J.A., van der Weide, R.H., Garcia-Nieto, A., Bravo, S., Ganji, M., Uit de Bos, J., Teunissen, H., Medema, R.H., de Wit, E., Haering, C.H., Dekker, C. and Rowland, B.D. (2019) Distinct roles for 
condensin's two ATPase sites in chromosome condensation. Mol. Cell, 76, 1-14.

Fang, Y. and Spector, D.L. (2005) Centromere positioning and dynamics in living Arabidopsis plants. Mol. Biol. Cell, 16, 5710-5718.

Fazzio, T.G. and Panning, B. (2010) Condensin complexes regulate mitotic progression and interphase chromatin structure in embryonic stem cells. J. Cell Biol., 188, 491-503.

Feng, S., Cokus, S.J., Schubert, V., Zhai, J., Pellegrini, M. and Jacobsen, S.E. (2014) Genome-wide Hi-C analyses in wild-type and mutants reveal high-resolution chromatin interactions in Arabidopsis. Mol. Cell, 55, 694-707.

Fransz, P., de Jong, J.H., Lysak, M., Castiglione, M.R. and Schubert, I. (2002) Interphase chromosomes in Arabidopsis are organized as well defined chromocenters from which euchromatin loops emanate. Proc. Natl. Acad. Sci., 99, 14584-14589.

Freeman, L., Aragon-Alcaide, L. and Strunnikov, A. (2000) The condensin complex governs chromosome condensation and mitotic transmission of rDNA. J. Cell Biol., 149, 811-824.

Fuchs, J., Demidov, D., Houben, A. and Schubert, I. (2006) Chromosomal histone modification patterns - from conservation to diversity. Trends Plant Sci., 11, 199-208.

Fujimoto, S., Yonemura, M., Matsunaga, S., Nakagawa, T., Uchiyama, S. and Fukui, K. (2005) Characterization and dynamic analysis of Arabidopsis condensin subunits, AtCAP-H and AtCAP-H2. Planta, 222, 293-300.

Gentry, M. and Hennig, L. (2014) Remodelling chromatin to shape development of plants. Exp. Cell Res., 321, 40-46.

George, C., Bozler, J., Nguyen, H. and Bosco, G. (2014) Condensins are required for maintenance of nuclear architecture. Cells, 3, 865-882.

Gerlich, D., Hirota, T., Koch, B., Peters, J.M. and Ellenberg, J. (2006) Condensin I stabilizes chromosomes mechanically through a dynamic interaction in live cells. Curr. Biol., 16, 333-344.

Ghavi-Helm, Y., Jankowski, A., Meiers, S., Viales, R., Korbel, J.O. and Furlong, E.M. (2019) Highly rearranged chromosomes reveal uncoupling between genome topology and gene expression. Nature Genetics, 51, 1272-1282.

Gibcus, J.H. and Dekker, J. (2013) The hierarchy of the 3D genome. Mol. Cell, 49, 773782.

Gibcus, J.H., Samejima, K., Goloborodko, A., Samejima, I., Naumova, N., Nuebler, J., Kanemaki, M.T., Xie, L., Paulson, J.R., Earnshaw, W.C., Mirny, L.A. and Dekker, J. (2018) A pathway for mitotic chromosome formation. Science, 359, 652.

Green, L.C., Kalitsis, P., Chang, T.M., Cipetic, M., Kim, J.H., Marshall, O., Turnbull, L., Whitchurch, C.B., Vagnarelli, P., Samejima, K., Earnshaw, W.C., Choo, K.H. and Hudson, D.F. (2012) Contrasting roles of condensin I and condensin II in mitotic chromosome formation. J. Cell Sci., 125, 1591-1604.

Haase, K., Macadangdang, J.K., Edrington, C.H., Cuerrier, C.M., Hadjiantoniou, S., Harden, J.L., Skerjanc, I.S. and Pelling, A.E. (2016) Extracellular forces cause the nucleus to deform in a highly controlled anisotropic manner. Sci. Rep., 6, 21300.

Habermann, F.A., Cremer, M., Walter, J., Kreth, G., von Hase, J., Bauer, K., Wienberg, J., Cremer, C., Cremer, T. and Solovei, I. (2001) Arrangements of macro- and microchromosomes in chicken cells. Chromosome Res., 9, 569-584.

Hartl, T.A., Sweeney, S.J., Knepler, P.J. and Bosco, G. (2008) Condensin II resolves chromosomal associations to enable anaphase I segregation in Drosophila male meiosis. PLoS Genet., 4, 18-22.

Heckmann, S., Lermontova, I., Berckmans, B., de Veylder, L., Bäumlein, H. and Schubert, I. (2011) The E2F transcription factor family regulates CENH3 expression in Arabidopsis thaliana. Plant J., 68, 646-656.

Herzog, S., Jaiswal, S.N., Urban, E., Riemer, A. Fischer, S. and Heidmann, S.K. (2013) 
Functional dissection of the Drosophila melanogaster condensin subunit Cap-G reveals its exclusive association with condensin I. PLoS Genet., 9, e1003463.

Hirano, T. (2012a) Condensins: universal organizers of chromosomes with diverse functions. Genes Dev., 26, 1659-1678.

Hirano, T. (2012b) Chromosome territories meet a condensin. PLoS Genet., 8, e1002939.

Hirota, T., Gerlich, D., Koch, B., Ellenberg, J. and Peters, J.M. (2004) Distinct functions of condensin I and II in mitotic chromosome assembly. J. Cell Sci., 117, 6435-6445.

Hotton, S.K. and Callis, J. (2008) Regulation of cullin RING ligases. Annu. Rev. Plant Biol., 59, 467-489.

Hudson, D.F., Vagnarelli, P., Gassmann, R. and Earnshaw, W.C. (2003) Condensin is required for nonhistone protein assembly and structural integrity of vertebrate mitotic chromosomes. Dev. Cell, 5, 323-336.

Iwasaki, O., Tanaka, A., Tanizawa, H., Grewal, S.I.S. and Noma, K.I. (2010) Centromeric localization of dispersed Pol III genes in fission yeast. Mol. Biol. Cell, 21, 254-265.

Jefferson, R.A., Kavanagh, T.A. and Bevan, M.W. (1987) GUS fusions: B-glucuronidase as a sensitive and versatile gene fusion marker in higher plants. EMBO J., 6, 3901-3907.

Jeppsson, K., Kanno, T., Shirahige, K. and Sjögren, C. (2014) The maintenance of chromosome structure: positioning and functioning of SMC complexes. Nat. Rev. Mol. Cell Biol., 15, 601-614.

Jost, K.L., Bertulat, B. and Cardoso, M.C. (2012) Heterochromatin and gene positioning: inside, outside, any side? Chromosoma, 121, 555-563.

Kagami, Y., Ono, M. and Yoshida, K. (2017) Plk1 phosphorylation of CAP-H2 triggers chromosome condensation by condensin II at the early phase of mitosis. Sci. Rep., 7, 5583.

Kikuchi, S., Kishii, M., Shimizu, M. and Tsujimoto, H. (2005) Centromere-specific repetitive sequences from Torenia, a model plant for interspecific fertilization, and whole-mount FISH of its interspecific hybrid embryos. Cytogenet. Genome Res., 109, 228-235.

Krause, M., Te Riet, J. and Wolf, K. (2013) Probing the compressibility of tumor cell nuclei by combined atomic force-confocal microscopy. Phys. Biol., 10, 065002.

Kudo, T., Sasaki, Y., Terashima, S., Matsuda-Imai, N., Takano, T., Saito, M., Kanno, M., Ozaki, S., Suwabe, K., Suzuki, G., Watanabe, M., Matsuoka, M., Takayama, S. and Yano, K. (2016) Identification of reference genes for quantitative expression analysis using large-scale RNA-seq data of Arabidopsis thaliana and model crop plants. Genes Genet. Syst., 91, 111-125.

Lau, A.C., Nabeshima, K. and Csankovszki, G. (2014) The C. elegans dosage compensation complex mediates interphase $\mathrm{X}$ chromosome compaction. Epigenetics Chromatin, 7, 31.

Layat, E., Sáez-Vásquez, J. and Tourmente, S. (2012) Regulation of pol I-transcribed 45S rDNA and pol III-transcribed 5S rDNA in Arabidopsis. Plant Cell Physiol., 53, 267-276.

Liu, C., Wang, C., Wang, G., Becker, C., Zaidem, M. and Weigel, D. (2016) Genome-wide analysis of chromatin packing in Arabidopsis thaliana at single-gene resolution. Genome Res., 26, 1057-1068.

Liu, C.M., McElver, J., Tzafrir, I., Joosen, R., Wittich, P., Patton, D., van Lammeren, A.A.M. and Meinke, D. (2002) Condensin and cohesin knockouts in Arabidopsis exhibit a titan seed phenotype. Plant J., 29, 405-415.

Livak, K.J. and Schmittgen, T.D. (2001) Analysis of relative gene expression data using real-time quantitative PCR and the $2-{ }^{\Delta \Delta \mathrm{C}}$ T method. Methods, 25, 402-408.

Longworth, M.S. Herr, A., Ji, J.Y. and Dyson, N.J. (2008) RBF1 promotes chromatin condensation through a conserved interaction with the Condensin II protein dCAP-D3. Genes Dev., 22, 1011-1024. 
Longworth, M.S., Walker, J.A., Anderssen, E., Moon, N.S., Gladden, A., Heck, M.M.S., Ramaswamy, S. and Dyson, N.J. (2012) A shared role for RBF1 and dCAP-D3 in the regulation of transcription with consequences for innate immunity. PLoS Genet., 8 , e1002618.

Love, M.I., Huber, W. and Anders, S. (2014) Moderated estimation of fold change and dispersion for RNA-seq data with DESeq2. Genome Biol., 15, 550.

Maeshima, K. and Laemmli, U.K. (2003) A two-step scaffolding model for mitotic chromosome assembly. Dev Cell 4: 467-480.

Maeshima, K., Tamura, S. and Shimamoto, Y. (2018) Chromatin as a nuclear spring. Biophys. Physicobiol., 15, 189-195.

Marenduzzo, D., Finan, K. and Cook, P.R. (2006) The depletion attraction: An underappreciated force driving cellular organization. J. Cell Biol., 175, 681-686.

Martinez-Zapater, J.M., Estelle, M.A. and Somerville, C.R. (1986) A highly repeated DNA sequence in Arabidopsis thaliana. Mol. Gen. Genet., 204, 417-423.

Mayer, R., Brero, A., von Hase, J., Schroeder, T., Cremer, T. and Dietzel, S. (2005) Common themes and cell type specific variations of higher order chromatin arrangements in the mouse. BMC Cell Biol., 6, 44.

Moissiard, G., Cokus, S.J., Cary, J., Feng, S., Billi, A.C., Stroud, H., Husmann, D., Zhan, Y., Lajoie, B.R., McCord, R.P., Hale, C.J., Feng, W., Michaels, S.D., Frand, A.R., Pellegrini, M., Dekker, J., Kim, J.K. and Jacobsen, S.E. (2012) MORC family ATPases required for heterochromatin condensation and gene silencing. Science, 336, 1448-1451.

Nakamura, S., Mano, S., Tanaka, Y., Ohnishi, M., Nakamori, C., Araki, M., Niwa, T., Nishimura, M., Kaminaka, H., Nakagawa, T., Sato, Y. and Ishiguro, S. (2010) Gateway binary vectors with the bialaphos resistance gene, bar, as a selection marker for plant transformation. Biosci. Biotechnol. Biochem., 74, 1315-1319.

Onn, I., Aono, N., Hirano, M. and Hirano, T. (2007) Reconstitution and subunit geometry of human condensin complexes. EMBO J., 26, 1024-1034.

Ono, T., Fang, Y., Spector, D.L. and Hirano, T. (2004) Spatial and temporal regulation of condensins I and II in mitotic chromosome assembly in human cells. Mol. Biol. Cell, 15, 3296-3308.

Ono, T., Losada, A., Hirano, M., Myers, M.P., Neuwald, A.F. and Hirano, T. (2003) Differential contributions of condensin I and condensin II to mitotic chromosome architecture in vertebrate cells. Cell, 115, 109-121.

Parra, G., Bradnam, K., Rose, A.B. and Korf, I. (2011) Comparative and functional analysis of intron-mediated enhancement signals reveals conserved features among plants. Nucleic Acids Res., 39, 5328-5337.

Pecinka, A., Schubert, V., Meister, A., Kreth, G., Klatte, M., Lysak, M.A., Fuchs, J. and Schubert, I. (2004) Chromosome territory arrangement and homologous pairing in nuclei of Arabidopsis thaliana are predominantly random except for NOR-bearing chromosomes. Chromosoma, 113, 258-269.

Perrella, G., Lopez-Vernaza, M.A., Carr, C., Sani, E., Gossele, V., Verduyn, C., Kellermeier, F., Hannah, M.A. and Amtmann, A. (2013) Histone deacetylase complex 1 expression level titrates plant growth and abscisic acid sensitivity in Arabidopsis. Plant Cell, 25, 3491-3505.

Poulet, A., Duc, C., Voisin, M., Desset, S., Tutois, S., Vanrobays, E., Benoit, M., Evans, D.E., Probst, A.V. and Tatout, C. (2017) The LINC complex contributes to heterochromatin organisation and transcriptional gene silencing in plants. J. Cell Sci., 130, 590-601.

Robellet, X., Fauque, L., Legros, P., Mollereau, E., Janczarski, S., Parrinello, H., Desvignes, J.P., Thevenin, M. and Bernard, P. (2014) A genetic screen for functional 
partners of condensin in fission yeast. G3 Genes|Genomes|Genetics, 4, 373-381.

Robson M.I., Ringel A.R., and Mundlos S. (2019) Regulatory landscaping: how enhancerpromoter communication is sculpted in 3D. Mol. Cell, 74, 1110-1122.

Rose, A.B., Elfersi, T., Parra, G. and Korf, I. (2008) Promoter-proximal introns in Arabidopsis thaliana are enriched in dispersed signals that elevate gene expression. Plant Cell, 20, 543-551.

Rosin, L.F., Nguyen, S.C. and Joyce, E.F. (2018) Condensin II drives large-scale folding and spatial partitioning of interphase chromosomes in Drosophila nuclei. PLoS Genet., 14, e1007393.

Probst, A.V. and Mittelsten-Scheid, O. (2015) Stress-induced structural changes in plant chromatin. Curr. Opin. Plant. Biol., 27, 8-16.

Sakamoto, T., Sugiyama, T., Yamashita, T. and Matsunaga, S. (2019) Plant condensin II is required for the correct spatial relationship between centromeres and rDNA arrays. Nucleus., 10, 116-125.

Sakamoto, Y. and Takagi, S. (2013) LITTLE NUCLEI 1 and 4 regulate nuclear morphology in Arabidopsis thaliana. Plant Cell Physiol., 54, 622-633.

Savvidou, E., Cobbe, N., Steffensen, S., Cotterill, S. and Heck, M.M.S. (2005) Drosophila CAP-D2 is required for condensin complex stability and resolution of sister chromatids. J. Cell Sci., 118, 2529-2543.

Schägger, H. and von Jagow, G. (1987) Tricine-sodium dodecyl sulfate-polyacrylamide gel electrophoresis for the separation of proteins in the range from 1 to $100 \mathrm{kDa}$. Anal. Biochem., 166, 368-379.

Schmiesing, J., Gregson, H., Zhou, S. and Yokomori, K. (2000) A human condensin complex containing hCAP-C-hCAP-E and CNAP1, a homolog of Xenopus XCAP-D2, colocalizes with phosphorylated histone $\mathrm{H} 3$ during the early stage of mitotic chromosome condensation. Mol. Cell. Biol., 20, 6996-7006.

Schneider, C.A., Rasband, W.S. and Eliceiri, K.W. (2012) NIH Image to ImageJ: 25 years of image analysis. Nat. Meth., 9, 671-675.

Schubert, V. (2009) SMC proteins and their multiple functions in higher plants. Cytogenet. Genome Res., 124, 202-214.

Schubert, V., Berr, A. and Meister, A. (2012) Interphase chromatin organisation in Arabidopsis nuclei: constraints versus randomness. Chromosoma, 121, 369-387.

Schubert, V., Lermontova, I. and Schubert, I. (2013) The Arabidopsis CAP-D proteins are required for correct chromatin organisation, growth and fertility. Chromosoma, 122, 517-533.

Shao, Y., Lu, N., Wu, Z., Cai, C., Wang, S., Zhang, L., Zhou, F., Xiao, S., Liu, L., Zeng, X., Zheng, H., Yang, C., Zhao, Z., Zhao, G., Zhou, J., Xue, X., Qin, Z. (2018) Creating a functional single-chromosome yeast. Nature, 560, 331-335.

Siddiqui, N.U., Stronghill, P.E., Dengler, R.E., Hasenkampf, A. and Riggs, C.D. (2003) Mutations in Arabidopsis condensin genes disrupt embryogenesis, meristem organization and segregation of homologous chromosomes during meiosis. Development, 130, 32833295.

Sieburth, L.E. and Meyerowitz, E.M. (1997) Molecular dissection of the AGAMOUS control region shows that cis elements for spatial regulation are located intragenically. Plant Cell, 9, 355-365.

Skibbens, R.V. (2019) Condensins and cohesins - one of these things is not like the other! $J$. Cell Sci., 132, jcs220491.

Skylar, A., Matsuwaka, S. and Wu, X. (2013) ELONGATA3 is required for shoot meristem cell cycle progression in Arabidopsis thaliana seedlings. Dev. Biol., 382, 436-445.

Smith, S.J., Osman, K. and Franklin, F.C.H. (2014) The condensin complexes play distinct roles to ensure normal chromosome morphogenesis during meiotic division in 
Arabidopsis. Plant J., 80, 255-268.

Soppe, W.J.J., Jasencakova, Z., Houben, A., Kakutani, T., Meister, A., Huang, M.S., Jacobsen, S.E., Schubert, I. and Fransz, P.F. (2002) DNA methylation controls histone H3 lysine 9 methylation and heterochromatin assembly in Arabidopsis. EMBO J., 21, 6549-6559.

Sparkes, I.A., Runions, J., Kearns, A. and Hawes, C. (2006) Rapid, transient expression of fluorescent fusion proteins in tobacco plants and generation of stably transformed plants. Nat. Protoc., 1, 2019-2025.

Stam M., Tark-Dame M. and Fransz P. (2019) 3D genome organization: a role for phase separation and loop extrusion? Curr. Opin. Plant Biol., 48, 36-46.

Sun, M., Biggs, R., Hornick, J. and J.F. Marko (2018) Condensin controls mitotic chromosome stiffness and stability without forming a structurally contiguous scaffold. Chromosome Res., 26, 277-295.

Szabo, Q., Bantignies, F. and Cavalli G. (2019) Principles of genome folding into topologically associating domains. Sci. $A d v, \mathbf{5}$, eaaw 1668 .

Szklarczyk, D., Gable, A.L., Lyon, D., Junge, A., Wyder, S., Huerta-Cepas, J., Simonovic, M., Doncheva, N.T., Morris, J.H., Bork, P., Jensen, L.J. and Mering, C.V. (2019) STRING v11: protein-protein association networks with increased coverage, supporting functional discovery in genome-wide experimental datasets. Nucleic Acids Res., 47, D607-D613.

Tatout, C., Evans, D.E., Vanrobays, E., Probst, A.V. and Graumann, K. (2014) The plant LINC complex at the nuclear envelope. Chromosom. Res., 22, 241-252.

Tessadori, F., Chupeau, M.C., Chupeau, Y., Knip, M., Germann, S., van Driel, R., Fransz, P. and Gaudin, V. (2007) Large-scale dissociation and sequential reassembly of pericentric heterochromatin in dedifferentiated Arabidopsis cells. J. Cell Sci., 120, 1200-1208.

Van Leene, J., Eeckhout, D., Cannoot, B., De Winne, N., Persiau, G., Van De Slijke, E., Vercruysse, L., Dedecker, M., Verkest, A., Vandepoele, K., Martens, L., Witters, E., Gevaert, K. and De Jaeger, G. (2014) An improved toolbox to unravel the plant cellular machinery by tandem affinity purification of Arabidopsis protein complexes. Nat. Protoc., 10, 169-187.

Van Leene, J., Eeckhout, D., Persiau, G., van de Slijke, E., Geerinck, J., van Isterdael, G., Witters, E. and de Jaeger, G. (2011) Isolation of transcription factor complexes from Arabidopsis cell suspension cultures by tandem affinity purification. In L. Yuan and S. E. Perry, eds. Plant transcription factors. Meth. Mol. Biol., 195-218.

Van Ruiten, M.S. and Rowland, B.D. (2018) SMC Complexes: universal DNA looping machines with distinct regulators. Trends Genet., 34, 477-487.

Van Zanten, M., Koini, M.A., Geyer, R., Liu, Y., Brambilla, V., Bartels, D., Koornneef, M., Fransz, P., Soppe and W.J. (2011) Seed maturation in Arabidopsis thaliana is characterized by nuclear size reduction and increased chromatin condensation. Proc. Natl. Acad. Sci. U S A, 108, 20219-20224.

Verlinden, L., Eelen, G., Beullens, I., Van Camp, M., Van Hummelen, P., Engelen, K., Van Hellemont, R., Marchal, K., De Moor, B., Foijer, F., Te Riele, H., Beullens, M., Bollen, M., Mathieu, C., Bouillon, R. and Verstuyf, A. (2005) Characterization of the condensin component Cnap1 and protein kinase melk as novel E2F target genes downregulated by 1,25-dihydroxyvitamin D3. J. Biol. Chem., 280, 37319-37330.

Wallace, H.A. and Bosco, G. (2013) Condensins and 3D organization of the interphase nucleus. Curr. Genet. Med. Rep., 1, 219-229.

Wallace, H.A., Klebba, J.E., Kusch, T., Rogers, G.C. and Bosco, G. (2015) Condensin II regulates interphase chromatin organization through the Mrg-binding motif of CapH2. G3 Genes/Genomes/Genetics 5, 803-817. 
Walther, N., Hossain, M.J., Politi, A.Z., Koch, B., Kueblbeck, M., Odegard-Fougner, O., Lampe, M. and Ellenberg, J. (2018) A quantitative map of human condensins provides new insights into mitotic chromosome architecture. J. Cell Biol., 217, 23092328

Wang, H., Dittmer, T.A. and Richards, E.J. (2013) Arabidopsis CROWDED NUCLEI (CRWN) proteins are required for nuclear size control and heterochromatin organization. BMC Plant Biol., 13, 200.

Wang, J., Blevins, T., Podicheti, R., Haag, J.R., Tan, E.H., Wang, F. and Pikaard, C.S. (2017) Mutation of Arabidopsis SMC4 identifies condensin as a corepressor of pericentromeric transposons and conditionally expressed genes. Genes Dev., 31, 16011614.

Wang, Z., Cao, H., Chen, F. and Liu, Y. (2014) The roles of histone acetylation in seed performance and plant development. Plant Physiol. Biochem., 84, 125-133.

Weisshart, K., Fuchs, J. and Schubert, V. (2016) Structured illumination microscopy (SIM) and photoactivated localization microscopy (PALM) to analyze the abundance and distribution of RNA polymerase II molecules on flow-sorted Arabidopsis nuclei. BioProtocol, 6. e1725.

Winter, D., Vinegar, B., Nahal, H., Ammar, R., Wilson, G.V. and Provart, N.J. (2007) An "Electronic Fluorescent Pictograph" browser for exploring and analyzing large-scale biological data sets. PLoS One, 8, e718.

Wu, T.D. and Nacu, S. (2010) Fast and SNP-tolerant detection of complex variants and splicing in short reads. Bioinformatics, 26, 873-881.

Xia, Y., Pfeifer, C.R., Cho, S., Discher, D.E. and Irianto, J. (2018) Nuclear mechanosensing. Emerg. Top. Life Sci., 2, 713-725.

Yilmaz, A., Mejia-Guerra, M.K., Kurz, K., Liang, X., Welch, L. and Grotewold E. (2011) AGRIS: the Arabidopsis gene regulatory information server, an update. Nucleic Acids Res., 39, D1118-D1122.

Yoo, S.D., Cho, Y.H. and Sheen, J. (2007) Arabidopsis mesophyll protoplasts: a versatile cell system for transient gene expression analysis. Nat. Protoc., 2, 1565-1572.

Yuen, K.C., Slaughter, B.D. and Gerton, J.L. (2017) Condensin II is anchored by TFIIIC and $\mathrm{H} 3 \mathrm{~K} 4 \mathrm{me} 3$ in the mammalian genome and supports the expression of active dense gene clusters. Sci. Adv., 3, e1700191.

Zamariola, L., de Storme, N., Vannerum, K., Vandepoele, K., Armstrong, S.J., Franklin, F.C.H. and Geelen, D. (2014) SHUGOSHINs and PATRONUS protect meiotic centromere cohesion in Arabidopsis thaliana. Plant J., 77, 782-794.

Zelkowski, M., Zelkowska, K., Conrad, U., Hesse, S., Lermontova, I., Marzec, M., Meister, A., Houben, A. and Schubert, V. (2019) Arabidopsis NSE4 proteins act in somatic nuclei and meiosis to ensure plant viability and fertility. Front. Plant Sci., 10, 774.

Zhang, T., Paulson, J.R., Bakhrebah, M., Kim, J.H., Nowell, C., Kalitsis, P. and Hudson, D.F. (2016) Condensin I and II behaviour in interphase nuclei and cells undergoing premature chromosome condensation. Chromosom. Res., 24, 243-269. 


\section{Figure legends}

Figure 1. A. thaliana condensin I and II subunit composition based on models of Nasmyth and Hearing (2005) and Schubert (2009). Both condensin complexes can be formed presumably by SMC4 and two alternative SMC2 subunits. Condensin I contains in addition CAP-D2, CAP-G and the $\gamma$-kleisin CAP-H, condensin II CAP-D3, CAP-G2 and the $\beta$-kleisin CAP-H2 (www.arabidopsis.org; Fujimoto et al., 2005). In the present work we confirm via analyzing CAP-D2 and CAP-D3 the interaction with the other respective subunits, and thus the presence of condensin I and II in $A$. thaliana.

Figure 2. Transcription of $C A P-D 2$ and $C A P-D 3$ in different tissues. Relative fold change expression (a) and relative expression (ddCT) (b) in flower buds, roots and rosette leaves compared to seedlings. The values were normalized to the geometric mean of the house keeping genes $P P 2 A$ and $R H I P 1$ and relative to the expression in seedlings. Lower ddCT values indicate higher transcription. Error bars in (b) represent the standard deviation between three biological replicates (each in triplicates). No error bars are shown in (a) since the fold change is a direct conversion of the ddCT values.

Figure 3. $\boldsymbol{C A P}$-D2 and $\boldsymbol{C A P}-D 3$ promoter activity. a) Schemata of the promoter regions, the first two introns and three exons of $C A P-D 2$ and $C A P-D 3$. The start of the coding region is marked by an arrow and the blue lines represent the position of the E2F binding sites. Below are shown the different tested promoter versions fused to the GUS gene. b) Histochemical GUS staining (blue staining) in root meristems, leaves, stipules (arrows; Pro5, 7) and apical meristems of plants transformed with the indicated promoter versions Pro5-9 and Pro10-11. Images of Pro4 are not depicted since no transformants could be isolated.

Figure 4. Localization of CAP-D2 in protoplasts of $A$. thaliana.

a) CAP-D2 is present in the cytoplasm and nuclei of leaf protoplasts. Untransformed protoplasts (negative control) and transformed with $C A P-D 2$ fused C-terminally to EYFP, and free EYFP (positive control) are presented. DAPI staining indicates the nuclei (arrows) and small signals in the cytoplasm corresponding to chloroplast DNA.

b) Western blot analysis on total protein extracts from protoplasts untransformed (negative C), transformed with free EYFP (positive C) or with CAP-D2-EYFPc. The detection was performed with anti-GFP antibodies or anti-CAP-D2 serum. The intense band of $27 \mathrm{kDa}$ (green arrow) corresponds to free EYFP. The bands of $187 \mathrm{kDa}$ (red arrow) correspond to the CAP-D2_EYFP fusion protein.

Figure 5. cap-d3 mutations impair centromere distribution, but not chromosome territory compaction. a) Gene structure model of CAP-D3. Red boxes represent exons, lines the introns and the lighter red box the 3'UTR. The T-DNA insertion sites of the cap-d3 SAIL and cap-d3 SALK lines are indicated. b) Rosette leave stage phenotypes of homozygous cap-d3 SAIL, cap-d3 SALK and wild-type (Wt) plants. c) Schematic representation of chromosome 1 and the localization of the chromosome territory 1 bottom (CT1B), and the centromeric pAL probe (labelling all ten centromeres present in the nuclei). d) SIM of a 4C nucleus labelled by FISH with the CT1B and pAL probes. e) Box plot diagram of the CT1B and the nucleus area sizes of cap-d3 SAIL, cap-d3 SALK and Wt nuclei. The boxes indicate upper and lower quartiles and the black bar the median. f) and g) pAL signal frequencies in 4C nuclei of cap-d3 SAIL, cap-d3 SALK, cap-d3 SALK complemented and Wt. $\mathrm{n}=$ total number of nuclei analyzed from two different plants in $\mathbf{e}$ ) and $\mathbf{f}$ ), and from three different plants in $\mathbf{g}$ ).

Figure 6. cap-d3 mutations induce of $45 S$ rDNA association, but do not influence $5 \mathrm{~S}$ rDNA and the spatial centromere arrangement in interphase nuclei. a) FISH signals of $45 \mathrm{~S}$ rDNA and $5 \mathrm{~S}$ rDNA on $4 \mathrm{C}$ nuclei of wild-type (Wt) and cap- $d 3$ SAIL mutants. The ideogram (right) represents the A. thaliana chromosomes showing the localization of $45 \mathrm{~S}$ and $5 \mathrm{~S}$ rDNA. b) and c) Frequency of $45 \mathrm{~S}$ and $5 \mathrm{~S}$ rDNA signals in $4 \mathrm{C}$ nuclei of Wt and the cap- $d 3$ mutants. $\mathrm{n}=$ total number of nuclei analyzed from three different plants. d) SIM orthogonal view of FISH with the centromeric repeat (pAL) on structurally preserved acrylamide-embedded nuclei of $\mathrm{Wt}$ and cap- $d 3$ mutants. Blue, green and red rectangles show $\mathrm{x}-\mathrm{y}, \mathrm{x}-\mathrm{z}$ and $\mathrm{y}-\mathrm{z}$ optical cross-sections, respectively. 
bioRxiv preprint doi: https://doi.org/10.1101/2019.12.12.873885; this version posted December 12, 2019. The copyright holder for this preprint (which was not certified by peer review) is the author/funder. All rights reserved. No reuse allowed without permission.

Figure 7. cap-d3 mutations do not modify the global epigenetic landscape in interphase nuclei. a) 5 -methyl-cytosine immunolocalization on $4 \mathrm{C}$ nuclei of wild-type $(\mathrm{Wt})$ and the cap- $d 3$ mutants. b) Southern blot analysis of the cap- $d 3$ mutants and Wt genomic DNA digested with HpaII (H) or MspI (M) and hybridized with the $\mathrm{P}^{32}$-labelled centromeric repeat $\mathrm{pAL}$ do not show different digestion patterns. c) Immunolocalization of histone $\mathrm{H} 3 \mathrm{~K} 4 \mathrm{me} 3$, H3K9me1 and $\mathrm{H} 3 \mathrm{~K} 14 \mathrm{ac}$ on $4 \mathrm{C}$ nuclei of $\mathrm{Wt}$ and the cap- $d 3$ mutants.

Figure 8. Transcriptome analysis of cap-d3 mutants and wild-type plantlets. a) Volcano plots showing transcriptome comparisons between cap-d3 SALK, cap-d3 SAIL and Wt. The horizontal dotted line corresponds to $\mathrm{pAdj}=0.05$. Genes below are depicted in black and above in grey. The red genes are differentially expressed (DEG) at a threshold of 2 fold change (i.e., up-regulated: $\geq 1 \log 2$ FC, or down-regulated: $\leq-1 \log 2$ FC) and with a pAdj $\leq 0.05$. pAdj is the p-value corrected for multiple testing with the Benjamini-Hochberg adjustment. b) Venn diagram showing the DEG across the three comparisons. Each circle comprises all the DEG genes of one comparison and the intersections between circles are the common DEG. For example: the blue circle represents the cap-d3 SAIL vs. Wt DEG, which are 398, of those: 83 are the same as in cap-d3 SALK vs. Wt, 53 are the same as in cap-d3 SAIL vs. cap-d3SALK, 3 are differentially expressed in all comparison and 259 are only present in cap-d3 SAIL vs. Wt. c) The ideogram of A. thaliana chromosomes showing the position of the 83 cap-d3 DEG along the chromosomes.

Figure 9. Model explaining the function of A. thaliana CAP-D3 in interphase nuclei. Two chromosomes are represented in blue and in red with euchromatin emanating loops from their pericentromeric chromocenters (rosette chromosome model; Fransz et al., 2002; de Nooijer et al., 2009). CAP-D3 (green circles) localizes along euchromatin creating the chromatin loops rigid to keep the chromocenters separated (left). In absence of CAP-D3 the chromatin loops are not stiff enough to counterbalance the depletion-attraction forces (Marenduzzo et al., 2006). Consequently, the chromocenters cluster (right).

\section{Supplementary Data}

Figure S1. In silico analysis of $A$. thaliana $C A P-D 2$ and $C A P-D 3$ expression. The results obtained with the Arabidopsis eFP Browser 2.0 (bar.utoronto.ca) revealed a similar expression level for both genes with high (red), medium (orange) and low (yellow) expression in different organs and developmental stages.

Figure S2. Protein-protein interaction network of CAP-D2 (condensin I) and CAP-D3 (condensin II). Both A. thaliana CAP-D2 (a, c) and CAP-D3 (b, c) proteins (red) interact potentially with the other coiled-coil condensin SMC complex components (green) and the condensin I- and condensin II-specific subunits (yellow). The network was generated by the STRING program (http://string-db.org/) analysis at scores $>0.90$ (a, b) and $>0.70$ (c), respectively. The black lines in between the proteins indicate the supporting evidence from experimental data available from different species. The dashed lines embrace the condensin I and II subunits in (c).

Figure S3. Affinity purified CAP-D2 and CAP-D3 GS-tagged. Coomasie staining of a SDS-PAGE gel with protein extracts from cells expressing CAP-D2-GS and CAP-D3-GS. The asterisks indicate the CAP-D2-GS (176 kDa) and CAP-D3-GS (163 kDa) proteins, respectively.

Figure S4. CAP-G and CAP-H fused to EYFPc localize in the nucleus (arrows) and cytoplasm of A. thaliana protoplasts. The dark regions are chloroplasts.

Figure S5. CAP-D2, CAP-H and CAP-G fused to EYFPc localize in the nucleus (arrows) and cytoplasm of $N$. benthamiana leaf epidermal cells

Figure S6. Western blot analysis confirms the correct size of the CAP-D2 recombinant protein (CAP-D2ct). Tested against anti-His-tag and anti-T7-tag the recombinant protein produced in E. coli has a the expected weight of $59.92 \mathrm{kDa}$ including the T7- and His-tags on the $\mathrm{N}$-t and C-termini, respectively. The arrow marks the band containing the CAP-D2_ct recombinant protein. 
Figure S7. Western blot on different amounts (1-100 ng) of the CAP-D2_ct recombinant protein against the anti-CAP-D2 serum indicates the high sensitivity of anti-CAP-D2.

Figure S8. Immunolocalization of histone modifications in cap-d3 mutants and wild-type plants. No differences were detected in $4 \mathrm{C}$ nuclei of wild-type (Wt) and the cap-d3 SAIL, cap-d3 SALK mutants tested with antibodies against histone H3K27me3 (euchromatic); H3K9me2 (heterochromatic); H3K9ac and with antibodies recognizing $\mathrm{H} 3 \mathrm{~K} 14+18+23+27 \mathrm{ac}$.

Table 1. Condensin subunits co-purifying with CAP-D2 and CAP-D3.

\begin{tabular}{|c|c|c|c|}
\hline Bait & Interactor (AGI) & Times detected & Average MASCOT Score \\
\hline CAP-D2-GS & CAP-D2 (AT3G57060) & 3 & 4539 \\
\hline CAP-D2-GS & SMC4 (AT5G48600) & 3 & 2543 \\
\hline CAP-D2-GS & SMC2A (AT5G62410) & 3 & 2497 \\
\hline CAP-D2-GS & SMC2B (AT3G47460) & 3 & 1932 \\
\hline CAP-D2-GS & CAP-G (AT5G37630) & 3 & 1421 \\
\hline CAP-D2-GS & CAP-H (AT2G32590) & 3 & 920 \\
\hline CAP-D3-GS & CAP-D3 (AT4G15890) & 3 & 6668 \\
\hline CAP-D3-GS & SMC4 (AT5G48600) & 3 & 1572 \\
\hline CAP-D3-GS & SMC2A (AT5G62410) & 3 & 819 \\
\hline CAP-D3-GS & CAP-G2 (AT1G64960) & 3 & 218 \\
\hline CAP-D3-GS & CAP-H2 (AT3G16730) & 3 & 206 \\
\hline CAP-D3-GS & SMC2B (AT3G47460) & 2 & 503 \\
\hline
\end{tabular}

Table 2. Gene ontology (GO) categories enriched in the 83 cap-d3 differentially expressed genes (DEGs). FDR (False Discovery Rate): p-value adjusted for multiple testing.

\begin{tabular}{lllr} 
Ontology & GO term & Description & FDR \\
\hline Biological process & GO:0009414 & Response to water deprivation & 0.0001 \\
Biological process & GO:0009415 & Response to water & 0.0001 \\
Biological process & GO:0042221 & Response to chemical stimulus & 0.0021 \\
Biological process & GO:0009628 & Response to abiotic stimulus & 0.0033 \\
Biological process & GO:0050896 & Response to stimulus & 0.01 \\
Biological process & GO:0006950 & Response to stress & 0.041 \\
Molecular function & GO:0030528 & Transcription regulator activity & 0.024 \\
Molecular function & GO:0003700 & Transcription factor activity & 0.047
\end{tabular}

Table S1. List of proteins co-purified with CAP-D2-GS. Only proteins present in the three affinity purifications and with no occurrence among the non-specific proteins are listed.

\begin{tabular}{lll} 
AGI & Protein name/function & Average MASCOT Score \\
\hline AT3G57060 & CAP-D2 & 4538.87 \\
AT5G48600 & SMC4 & 2542.57 \\
AT5G62410 & SMC2A & 2497.43 \\
AT3G47460 & SMC2B & 1932.23 \\
AT5G37630 & CAP-G & 1421.20 \\
AT2G32590 & CAP-H & 919.63 \\
AT3G46740 & protein TOC75-3 & 641.57
\end{tabular}


AT3G08943

AT1G72560

AT5G09840

AT2G20800

AT2G02090

AT5G64270

AT4G24840

AT1G07810

AT4G01100

AT3G60860

AT4G19490

AT3G54110

AT2G40730

AT4G02510

AT3G01280

AT4G05020

AT5G41950

AT5G16930

AT4G01400

AT4G02570

AT5G16210

AT2G39260

AT5G22770

AT5G18420

AT2G01690

AT3G45190

AT4G33650

AT4G02350

AT2G36200

AT5G26760

AT1G04080

AT3G62360

AT1G60200

AT4G32050

AT1G22730

AT5G13850

AT3G55410

AT2G18330

AT3G16830

AT2G22300

AT2G14120

AT4G01990

AT3G11710

AT5G49830

AT2G27170

AT1G48900

AT1G60070 armadillo/beta-catenin-like repeat-containing protein

551.70

PAUSED

476.33

putative endonuclease or glycosyl hydrolase

470.07

$\mathrm{NAD}(\mathrm{P}) \mathrm{H}$ dehydrogenase $\mathrm{B} 4$

445.47

CHR19 Chromatin remodelling 19

400.83

putative splicing factor

393.70

389.23

386.87

380.70

374.00

360.83

352.93

uncoupling mitochondrial protein 1

352.93

352.83

341.17

340.97

334.20

332.53

329.23

318.60

315.07

301.20

269.63

265.63

265.13

256.73

253.40

250.40

247.90

246.07

243.80

242.80

241.90

236.77

234.23

226.80

226.57

218.97

218.27

218.23

213.63

204.30

203.83

202.50

197.60

196.17

195.77

194.00 
AT2G27900

AT1G63810

AT4G21150

AT2G31810

AT4G27500

AT1G73430

AT1G71270

AT5G19760

AT3G54540

AT5G13110

AT5G08550

AT5G47480

AT5G65460

AT5G14580

AT5G09420

AT3G11400

AT5G08450

AT5G11980

AT1G79940

AT3G45970

AT2G42710

AT3G08030

AT5G18620

AT5G50320

AT1G31780

AT5G19400

AT5G10470

AT1G61040

AT1G67930

AT4G39690

AT1G03860

AT1G19870

AT3G16620

AT5G61970

AT1G32380

AT1G78380

AT3G59020

AT4G10320

AT4G24550

AT1G62740

AT1G11910

AT5G67500

AT5G46750

AT2G19480

AT5G66680

AT5G03540

AT5G42960 coiled-coil protein

193.10

nucleolar protein

192.43

ribophorin II (RPN2) family protein

187.57

ACT domain-containing small subunit of acetolactate synthase protein

186.10

proton pump interactor 1

186.07

putative conserved oligomeric golgi complex 3

182.73

178.03

176.33

175.57

171.50

167.73

167.73

167.23

163.70

162.17

155.40

153.20

153.10

152.73

152.73

147.03

146.87

146.83

146.53

144.90

142.17

138.43

137.57

136.70

136.10

135.43

135.23

133.90

133.60

128.47

128.00

125.70

121.20

121.07

117.43

117.33

116.70

115.70

115.53

114.07

112.07

110.17 


$\begin{array}{lll}\text { AT3G46220 } & \text { E3 UFM1-protein ligase 1-like protein } & 109.93 \\ \text { AT2G27030 } & \text { calmodulin 5 } & 109.70 \\ \text { AT3G44330 } & \text { M28 Zn-peptidase nicastrin } & 109.40 \\ \text { AT3G23300 } & \text { S-adenosyl-Lmethionine-dependent methyltransferases superfamily protein } & 107.67 \\ \text { AT1G49040 } & \text { stomatal cytokinesis defective (SCD1) } & 106.07 \\ \text { AT2G05120 } & \text { nucleoporin, Nup133/Nup155-like protein } & 103.40\end{array}$

Table S2. List of proteins co-purified with CAP-D3-GS. Only proteins present in the three affinity purifications and with no occurrence among the non-specific proteins are listed.

\begin{tabular}{|c|c|c|}
\hline AGI & Protein names/function & $\begin{array}{l}\text { Average MASCOT } \\
\text { Score }\end{array}$ \\
\hline AT4G15890 & CAP-D3 & 6668.17 \\
\hline AT5G48600 & SMC4 & 1571.70 \\
\hline AT5G62410 & SMC2A & 819.37 \\
\hline AT2G19480 & nucleosome assembly protein $1 ; 2$ & 496.57 \\
\hline AT2G38770 & intron-binding protein aquarius & 440.83 \\
\hline AT3G13290 & varicose-related protein & 416.07 \\
\hline AT4G02510 & translocase of chloroplast 159 & 407.33 \\
\hline AT3G54110 & uncoupling mitochondrial protein 1 & 399.83 \\
\hline AT1G48900 & signal recognition particle subunit SRP54 & 399.30 \\
\hline AT4G26110 & nucleosome assembly protein 1 -like 1 & 397.30 \\
\hline AT4G01990 & pentatricopeptide repeat-containing protein & 371.23 \\
\hline AT4G32050 & Neurochondrin family protein & 331.57 \\
\hline AT2G03510 & SPFH/Band 7/PHB domain-containing membrane-associated protein & 317.63 \\
\hline AT4G01100 & adenine nucleotide transporter 1 & 309.07 \\
\hline AT3G02200 & proteasome component $(\mathrm{PCI})$ domain protein & 308.57 \\
\hline AT2G42710 & ribosomal protein $.1 / \mathrm{L} 10$ family protein & 304.23 \\
\hline AT3G60860 & SEC7-like guanine nucleotide exchange family protein & 303.10 \\
\hline AT3G01280 & mitochondrial outer membrane protein porin 1 & 299.57 \\
\hline AT5G09840 & putative endonuclease or glycosyl hydrolase & 288.67 \\
\hline AT4G21150 & ribophorin II (RPN2) family protein & 250.40 \\
\hline AT5G18420 & CCR4-NOT transcription complex subunit & 245.07 \\
\hline AT1G64960 & CAP-G2 & 218.27 \\
\hline AT4G33510 & phospho-2-dehydro-3-deoxyheptonate aldolase 2 & 211.13 \\
\hline AT5G58410 & HEAT/U-box domain-containing protein & 210.40 \\
\hline AT5G19760 & mitochondrial substrate carrier family protein & 207.50 \\
\hline AT3G16730 & $\mathrm{CAP}-\mathrm{H} 2$ & 205.97 \\
\hline AT1G14850 & nucleoporin 155 & 200.63 \\
\hline AT1G06530 & tropomyosin-related protein & 198.07 \\
\hline AT5G40770 & prohibitin 3 & 194.70 \\
\hline AT3G02650 & pentatricopeptide repeat-containing protein & 186.60 \\
\hline AT2G20800 & NAD(P)H dehydrogenase $\mathrm{B} 4$ & 184.30 \\
\hline AT1G20960 & putative U5 small nuclear ribonucleoprotein helicase & 183.03 \\
\hline AT2G45140 & VAP-like protein 12 & 182.27 \\
\hline AT3G44330 & M28 Zn-peptidase nicastrin & 180.40 \\
\hline AT5G13110 & glucose-6-phosphate 1-dehydrogenase & 177.80 \\
\hline
\end{tabular}


AT4G24550

AT5G64270

AT5G66680

AT3G02090

AT2G39260

AT5G07340

AT2G33040

AT4G02150

AT5G50320

AT3G55620

AT5G27970

AT1G26460

AT1G55890

AT1G02370

AT5G11980

AT5G15610

AT5G20490

AT5G30510

AT4G17330

AT5G15020

AT2G20360

AT2G31810

AT1G64880

AT5G16930

AT1G27090

AT1G71410

AT3G55005

AT3G53130

AT3G49080

AT2G43950

AT2G26890

AT5G12470

AT4G31810

AT3G46950

AT3G18790

AT1G23280

AT1G67140

AT5G46750

AT3G61140

AT2G46020

AT4G21800

AT2G15630

AT4G38600

AT2G40890

AT4G11260

AT1G50030

AT4G05020
AP-4 complex subunit mu-1

174.97

putative splicing factor

170.03

putative dolichyl-di-phosphooligosaccharide-protein glycotransferase

168.17

putative mitochondrial processing peptidase

162.33

regulator of nonsense transcripts UPF2

161.90

calnexin homolog

155.57

155.37

155.03

154.50

153.50

153.37

153.33

152.80

151.40

148.23

146.13

146.00

145.93

144.13

142.40

141.63

140.57

139.73

134.10

132.70

132.50

129.97

129.13

128.83

128.77

126.27

125.97

122.43

119.33

116.20

114.93

113.27

110.80

110.77

109.03

108.77

108.30

108.17

103.80

103.67

102.90

101.63 


$\begin{array}{lll}\text { AT2G16485 } & \text { NERD (Needed for RDR2-independent DNA methylation) } & 101.37 \\ \text { AT2G16640 } & \text { putative chloroplast outer membrane protein } & 100.20\end{array}$

Table S3. Differentially expressed genes (DEGs) in the cap-d3 mutants. List of DEGs common to cap-d3 SAIL vs. wild-type (Wt) and cap-d3 SALK vs. Wt. Genes in bold are transcription factors.

\begin{tabular}{|c|c|c|c|}
\hline AGI & Protein name/function & $\begin{array}{l}\text { Log2 fold-change } \\
\text { SAIL vs. Wt }\end{array}$ & $\begin{array}{l}\text { Log2 fold- } \\
\text { change } \\
\text { SALK vs. Wt }\end{array}$ \\
\hline AT3G48360 & $\mathrm{Bt} 2$ & 3.20 & 2.68 \\
\hline AT1G50040 & Unknown & 3.17 & 2.57 \\
\hline AT5G25240 & Unknown & 2.32 & 1.52 \\
\hline AT2G28120 & Otu1 & 2.30 & 1.78 \\
\hline AT5G67480 & $\mathrm{Bt} 4$ & 2.22 & 1.51 \\
\hline AT5G05440 & PYL5 & 2.07 & 1.21 \\
\hline AT1G11260 & STP1 & 2.01 & 1.16 \\
\hline AT4G36850 & PQ-loop repeat family protein & 1.97 & 1.28 \\
\hline AT2G44910 & АTHB-4 & 1.90 & 1.64 \\
\hline AT1G32170 & ХTH30 & 1.90 & 1.04 \\
\hline AT3G23550 & DTX18 (LAL5) & 1.87 & 1.28 \\
\hline AT1G02380 & Unknown & 1.86 & 1.24 \\
\hline AT2G20670 & Unknown & 1.84 & 1.68 \\
\hline AT5G14120 & Major facilitator? & 1.80 & 1.40 \\
\hline AT3G15630 & Unknown & 1.80 & 1.17 \\
\hline AT2G42870 & PAR1(HLH1) & 1.78 & 1.12 \\
\hline AT2G47440 & Tetraticopeptide repeat & 1.75 & 1.62 \\
\hline AT1G02610 & RING/PHD zinc finger superfam. Prot? & 1.74 & 1.14 \\
\hline AT5G22920 & CHYR1 (ATRZPF34) & 1.68 & 1.12 \\
\hline AT5G19120 & Eukaryotic aspartyl protease family protein? & 1.67 & 1.64 \\
\hline AT2G25200 & $\begin{array}{l}\text { Unknown } \\
\text { XTH25, XYLOGLUCAN }\end{array}$ & 1.62 & 1.30 \\
\hline AT5G57550 & ENDOTRANSGLUCOSYLASE/HYDROLASE 25 & 1.62 & 1.13 \\
\hline AT3G30122 & $\begin{array}{l}\text { Pseudogene } \\
\text { OFP15, ARABIDOPSIS THALIANA OVATE FAMILY }\end{array}$ & 1.62 & 1.92 \\
\hline AT2G36050 & PROTEIN 15 & 1.62 & 1.37 \\
\hline AT2G18700 & TPS11, TREHALOSE PHOSPHATASE/SYNTHASE 11 & 1.62 & 1.10 \\
\hline AT4G17470 & Lipid methabolism & 1.56 & 1.09 \\
\hline AT2G15880 & Leucine rich repeat & 1.56 & 1.16 \\
\hline AT5G19190 & Unknown & 1.53 & 1.20 \\
\hline AT4G17460 & HAT1/JAB/JAIBA & 1.52 & 1.63 \\
\hline AT3G19680 & Unknown & 1.52 & 1.51 \\
\hline AT4G38470 & STY46 & 1.51 & 1.03 \\
\hline AT3G15450 & SEN5 & 1.45 & 1.10 \\
\hline AT1G69570 & Hipoxia induced TF & 1.45 & 1.01 \\
\hline AT5G35777 & Transposable element gene & 1.38 & 1.30 \\
\hline AT2G17230 & EXL5 & 1.35 & 1.08 \\
\hline AT2G20835 & Unknown & 1.31 & 1.12 \\
\hline AT2G22770 & NAI1 & 1.30 & 1.26 \\
\hline AT2G17740 & VGL & 1.28 & 1.59 \\
\hline AT5G28145 & Transposable element gene & 1.27 & 1.03 \\
\hline
\end{tabular}




\begin{tabular}{|c|c|c|c|}
\hline AT5G61590 & DEWAX & 1.25 & 1.04 \\
\hline AT4G14680 & APS3/ATPS3 & 1.25 & 1.25 \\
\hline AT1G70290 & ATTPS8, ATTPSC, TPS8 & 1.24 & 1.10 \\
\hline AT5G06870 & ATPGIP2, PGIP2 & 1.23 & 1.12 \\
\hline AT1G64200 & VHA-E3 & 1.22 & 1.02 \\
\hline AT3G53232 & DEVIL 20, DVL20, ROTUNDIFOLIA LIKE 1, RTFL1 & 1.21 & 1.13 \\
\hline AT2G23130 & AGP17 & 1.20 & 1.16 \\
\hline AT3G25760 & AOC1/ERD12 & 1.18 & 1.01 \\
\hline AT5G05600 & DOX & 1.17 & 1.11 \\
\hline AT1G70700 & JAZ9, TIFY7 & 1.17 & 1.33 \\
\hline AT5G56550 & OXS3 & 1.13 & 1.22 \\
\hline AT3G15500 & ANAC55 & 1.13 & 1.12 \\
\hline AT1G36370 & MSA1/SHM7 & 1.11 & 1.39 \\
\hline AT4G27410 & ANAC72/RD26 & 1.09 & $\mathbf{1 . 3 5}$ \\
\hline AT4G24015 & RHA4A & 1.09 & 1.05 \\
\hline AT3G49580 & LSU1 & 1.05 & 1.11 \\
\hline AT1G15125 & S-adenosyl-L-methionine-dependent methyltransferase & 1.04 & 1.03 \\
\hline AT4G10910 & Unknown & 1.03 & 1.14 \\
\hline AT5G63130 & Octicosapeptide/Phox/Bem1p family protein & -1.01 & -1.21 \\
\hline AT1G60190 & PUB19 & -1.06 & -1.07 \\
\hline AT3G62260 & Protein phosphatase $2 \mathrm{C}$ family protein & -1.09 & -1.07 \\
\hline AT5G15190 & Unknown & -1.09 & -1.15 \\
\hline AT4G25490 & ATCBF1/ DRE BINDING PROTEIN 1B & -1.09 & -1.07 \\
\hline AT3G21150 & ATBBX32 & -1.15 & -1.05 \\
\hline AT3G49710 & Pentatricopeptide repeat (PPR) superfamily protein & -1.17 & -1.28 \\
\hline AT2G34655 & Unknown & -1.19 & -1.04 \\
\hline AT5G05410 & DREB2 & -1.19 & -1.19 \\
\hline AT3G44450 & BIC-1 & -1.21 & -1.08 \\
\hline AT3G22540 & Unknown & -1.23 & -1.01 \\
\hline AT2G34650 & PID/ABR & -1.24 & -1.07 \\
\hline AT3G27210 & Unknown & -1.28 & -1.35 \\
\hline AT2G40750 & ATWRKY54 & -1.29 & -1.17 \\
\hline AT5G41400 & RING/U-box superfamily protein & -1.29 & -1.02 \\
\hline AT5G38005 & Unknown & -1.32 & -1.08 \\
\hline AT1G53080 & Legume lectin family protein & -1.35 & -1.20 \\
\hline AT3G22840 & ELIP & -1.36 & -1.21 \\
\hline AT4G04223 & Unknown & -1.37 & -1.83 \\
\hline AT5G49480 & $\mathrm{CP} 1$ & -1.42 & -1.12 \\
\hline AT2G01670 & NUDT17 & -1.48 & -1.58 \\
\hline AT4G25480 & ATCBF3/DREB1A & -1.77 & -1.19 \\
\hline AT1G70640 & octicosapeptide/Phox/Bem1p (PB1) domain-containing protein & -1.84 & -1.36 \\
\hline AT4G15248 & BBX30 & -1.96 & -1.00 \\
\hline $\begin{array}{l}\text { AT3G44990 } \\
\text { AT3G15310 }\end{array}$ & $\begin{array}{l}\text { XTH31,XTR } 8 \\
\text { Transposable element gene }\end{array}$ & $\begin{array}{l}-2.25 \\
-2.32\end{array}$ & $\begin{array}{l}-1.25 \\
-1.64\end{array}$ \\
\hline
\end{tabular}


Table S4. Primer sequences and usage. Linker sequences are in lower case letters, restriction sites are underlined and genomic sequences are written in upper case.

Primer use

cap-d3 SALK line genotyping

cap-d3 SALK line genotyping

cap-d3 SAIL line genotyping

cap-d3 SAIL line genotyping

T-DNA primer SAIL lines

T-DNA primer SALK lines

Transcript quantification

Transcript quantification

Transcript quantification

Transcript quantification

Transcript quantification

Transcript quantification

Transcript quantification

Transcript quantification

$C A P-G$ cloning into pEntry

$C A P-G$ cloning into pEntry

$C A P-H$ cloning into pEntry

$C A P-H$ cloning into pEntry

CAP-D2 Ct cloning into pET23a

CAP-D2 Ct cloning into pET23a

$C A P-D 2$ promotor cloning into pEntry

$C A P-D 2$ promotor cloning into pEntry

$C A P-D 2$ promotor cloning into pEntry

$C A P-D 2$ promotor cloning into pEntry

$C A P-D 2$ promotor cloning into pEntry

$C A P-D 3$ promotor cloning into pEntry

$C A P-D 3$ promotor cloning into pEntry

$C A P-D 3$ promotor cloning into pEntry
Primer name

SALK_094776

SALK_094776

SAIL_826B06

SAIL_826B06

SAIL_LB3

LBb1.3

D2QRT2_F

D2QRT2_R

D3QRT1_F

D3QRT1_R

PP2A_F

PP2A_R

At4G26410_F

At4G26410_R

CAPG_pEnt_f

CAPG_pEnt_r

CAPH_pentry_f

CAPH_pentry_r

D2CtSalI_F

D2CtNotI_R

D2-392F

D2-1156F

D2ProR

D2Int1R

D2Int2R

D3-474F

D3-1318F

D3ProR
Sequence 5'-3'

TGGTTTGAAAATGGTTGCTTC

AGCGATAGAAGGAATCGAAGG

TGAAGAAGGTGGATTTGATGC

CGGAAATAGCTGAAACTGCAG

AGCATCTGAATTTCATAACCAATCTCGATACAC

ATTTTGCCGATTTCGGAAC

CCACCCAAGAGAACAATGGC

TGCACACTCCCCAATCAGAT

AGAATGACGTACAAGGGCTAGA

ATCGCCAGCCCATGTAGAAG

TAACGTGGCCAAAATGATGC

GTTCTCCACAACCGCTTGGT

GAGCTGAAGTGGCTTCCATGAC

GGTCCGACATACCCATGATCC

acgtGTCGACATGGGCGAAGAATCAGAAATC

attaGCGGCCGCgaTTCATCTGAATCATCTGCTGT

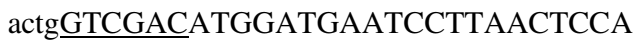

attaGCGGCCGCagGGCAAGGTGTATTGTTAGATCA

actGTCGACtaGGTTCTGTTGAGAAGAATCTG

tattGCGGCCGCACTTCTACTTCCTGACCT

gtgcGTCGACCTCAAAGCTTTTCTGCTTC

gtgcGTCGACTGGTACTGAAGCTAAGAAGG

gaagGCGGCCGCTTTTTCTAGAGAGAGAGAGA

caatGCGGCCGCTCAGAAAGGTCAAAGGATAC

aaatGCGGCCGCTTTTTCCTCCCTCGTGCTG

gtgcGTCGACATTTTGTTGTCTAGAATTTG

gtgcGTCGACTTTTCCTCTGTTCAATAG

taatGCGGCCGCGGCGATTCTCTACTGATAGA 
Table S5. Antibodies and their dilutions used for immunolocalization.

\begin{tabular}{lll} 
Antibody name & Dilution used & \multicolumn{1}{c}{ Reference } \\
\hline Anti-CAP-D3 & $1: 200$ & Schubert et al. 2013 \\
Anti-CAP-D2 serum & $1: 200$ & This study \\
Anti-H3K27me3 & $1: 100$ & Merck 07-449 \\
Anti-H3K9me1 & $1: 200$ & Merck 07-395 \\
Anti-H3K9me2 & $1: 100$ & Merck 07-441 \\
Anti-H3K4me3 & $1: 200$ & Merck 07-473 \\
& $1: 500$ & Nobus Biological NBP2- \\
Anti-H3K9ac & & 44095 \\
Anti-H3K14ac & $1: 1000$ & Merck 07-353 \\
Anti-H3K18ac & $1: 1500$ & Abcam ab1191 \\
Anti-H3K9+14+18+23+27 & $1: 500$ & \\
ac & & Abcam ab47915 \\
Anti-5methylcytosine & $1: 100$ & Abcam ab10805 \\
Anti-GFP 488 & $1: 1000$ & Rockland 200-341-215 \\
Anti-Rabbit 488 & $1: 100$ & Dianova 711-545-152 \\
Anti-Rabbit Rhodamine & $1: 300$ & Jackson 111-025-003 \\
Anti Mouse 488 & $1: 50$ & Molecular probes A11001 \\
Anti Guinea Pig 488 & $1: 100$ & Molecular probes A11073
\end{tabular}




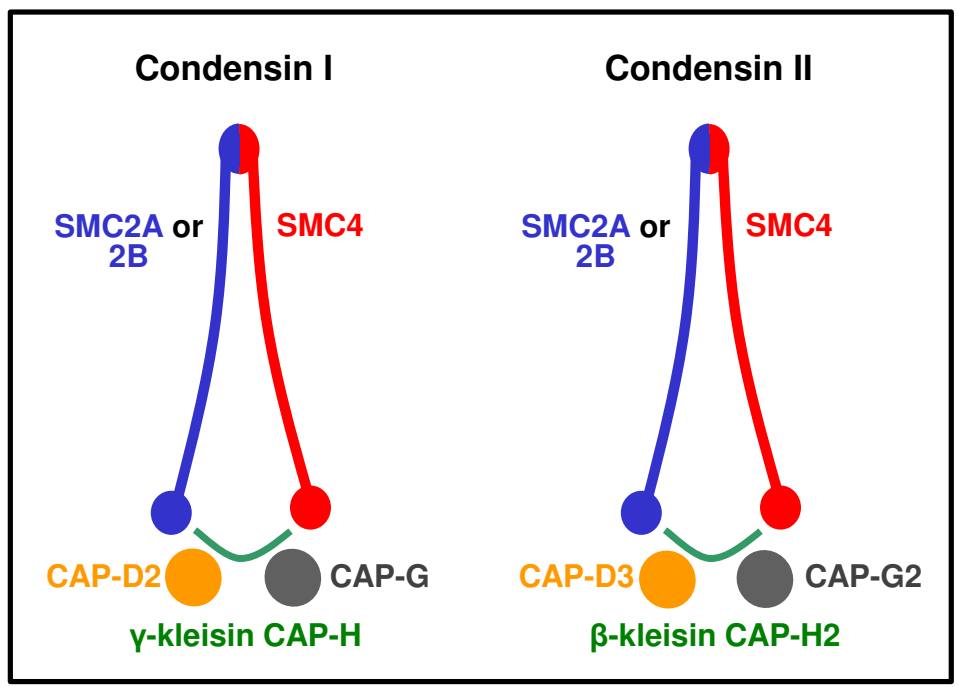

Figure 1. A. thaliana condensin I and II subunit composition based on models of Nasmyth and Hearing (2005) and Schubert (2009). Both condensin complexes can be formed presumably by SMC4 and two alternative SMC2 subunits. Condensin I contains in addition CAP-D2, CAP-G and the $\gamma$-kleisin CAP-H, condensin II CAP-D3, CAP-G2 and the $\beta$-kleisin CAP-H2 (www.arabidopsis.org; Fujimoto et al., 2005). In the present work we confirm via analyzing CAP-D2 and CAP-D3 the interaction with the other respective subunits, and thus the presence of condensin I and II in A. thaliana. 

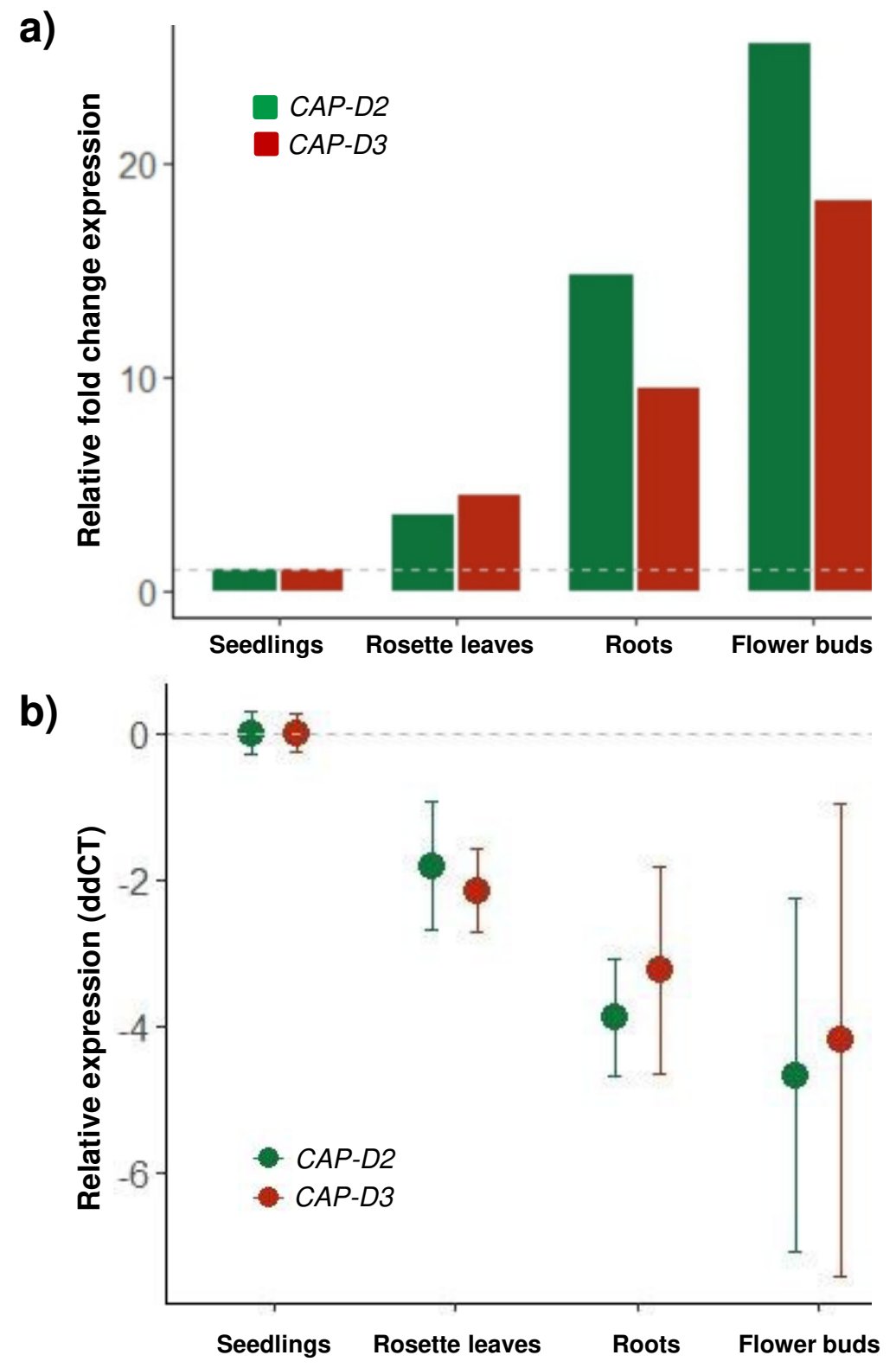

Figure 2. Transcription of $C A P-D 2$ and $C A P-D 3$ in different tissues. Relative fold change expression (a) and relative expression (ddCT) (b) in flower buds, roots and rosette leaves compared to seedlings. The values were normalized to the geometric mean of the house keeping genes PP2A and RHIP1 and relative to the expression in seedlings. Lower ddCT values indicate higher transcription. Error bars in (b) represent the standard deviation between three biological replicates (each in triplicates). No error bars are shown in (a) since the fold change is a direct conversion of the ddCTvalues. 
a)
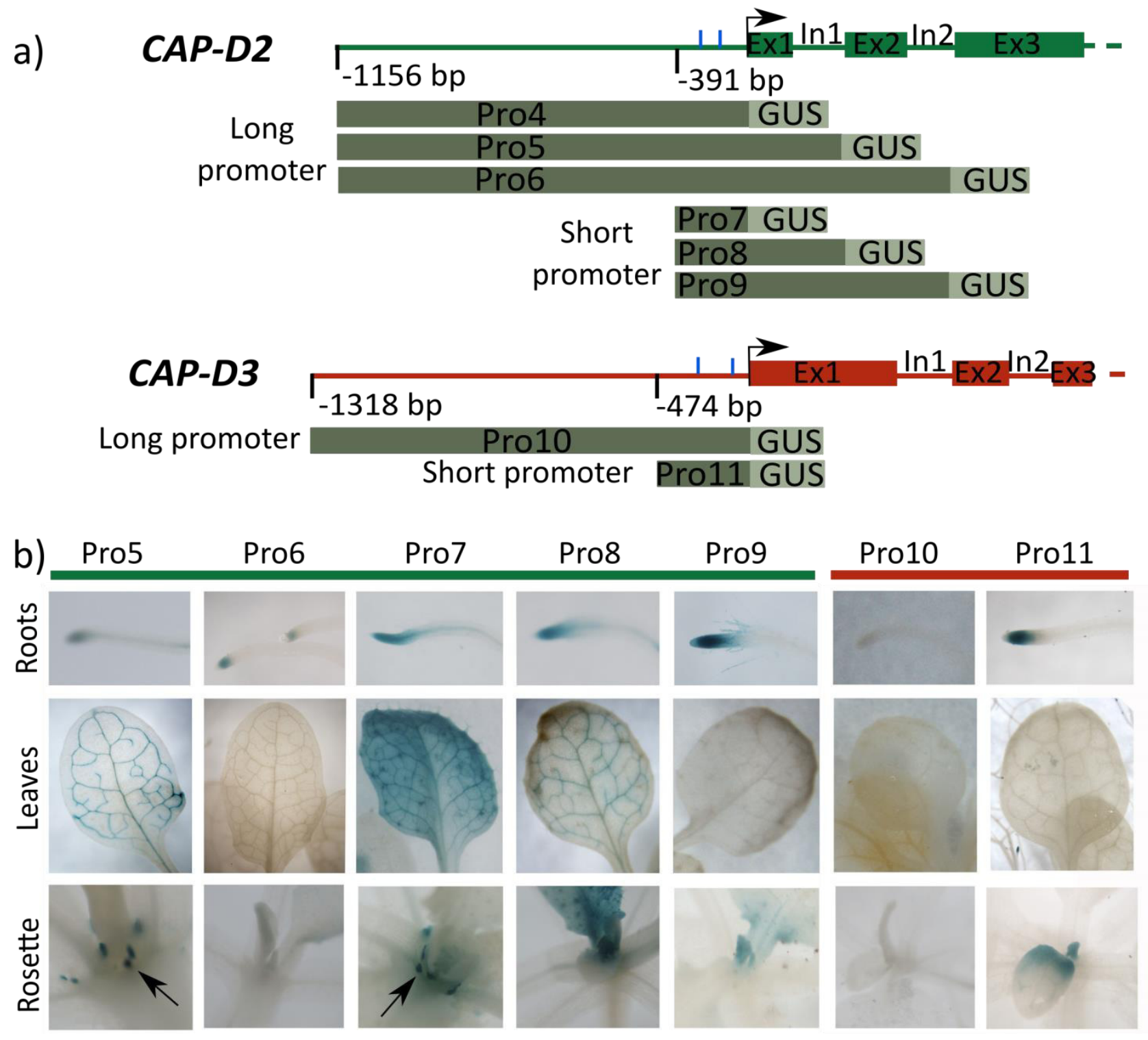

Figure 3. CAP-D2 and CAP-D3 promoter activity. a) Schemata of the promoter regions, the first two introns and three exons of $C A P-D 2$ and $C A P-D 3$. The start of the coding region is marked by an arrow and the blue lines represent the position of the E2F binding sites. Below are shown the different tested promoter versions fused to the GUS gene. b) Histochemical GUS staining (blue staining) in root meristems, leaves, stipules (arrows; Pro5, 7) and apical meristems of plants transformed with the indicated promoter versions Pro5-9 and Pro10-11. Images of Pro4 are not depicted since no transformants could be isolated. 

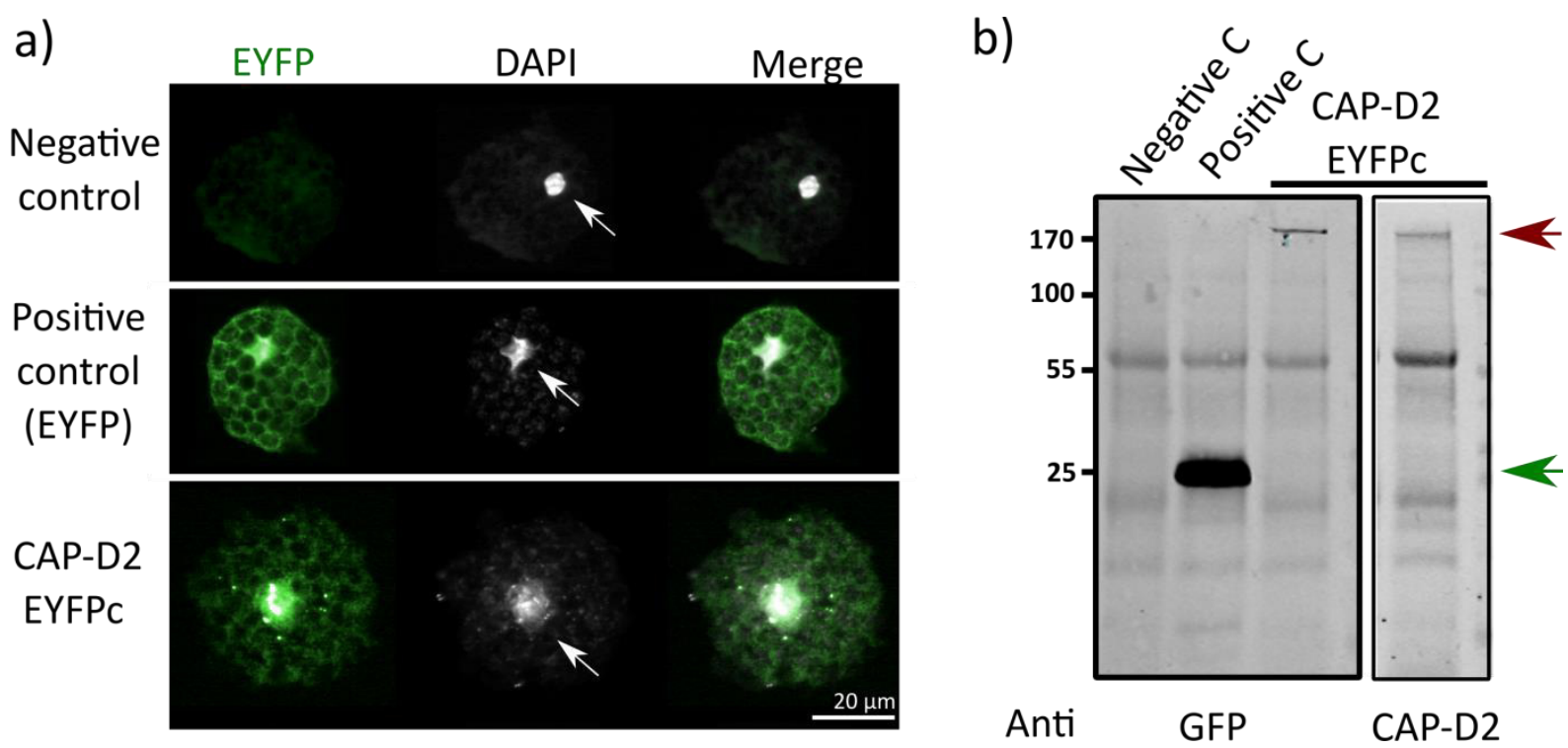

Figure 4. Localization of CAP-D2 in protoplasts.

a) CAP-D2 is present in the cytoplasm and nuclei of leaf protoplasts. Untransformed protoplasts (negative control) and transformed with CAP-D2 fused C-terminally to EYFP, and free EYFP (positive control) are presented. DAPI staining indicates the nuclei (arrows) and small signals in the cytoplasm corresponding to chloroplast DNA.

b) Western blot analysis on total protein extracts from protoplasts untransformed (negative C), transformed with free EYFP (positive C) or with CAP-D2-EYFPc. The detection was performed with anti-GFP antibodies or anti-CAP-D2 serum. The intense band of $27 \mathrm{kDa}$ (green arrow) corresponds to free EYFP. The bands of $187 \mathrm{kDa}$ (red arrow) correspond to the CAP-D2_EYFP fusion protein. 
a) $C A P-D 3$ At4g15890

b)

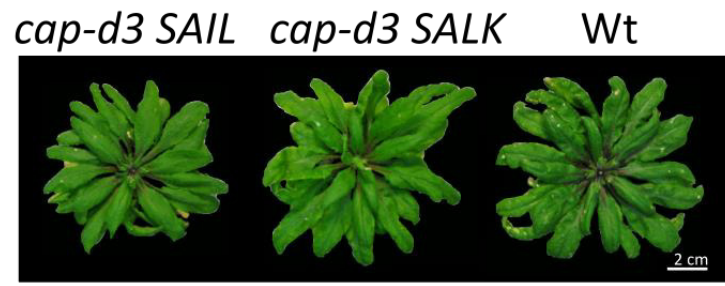

d)

d) DAPI

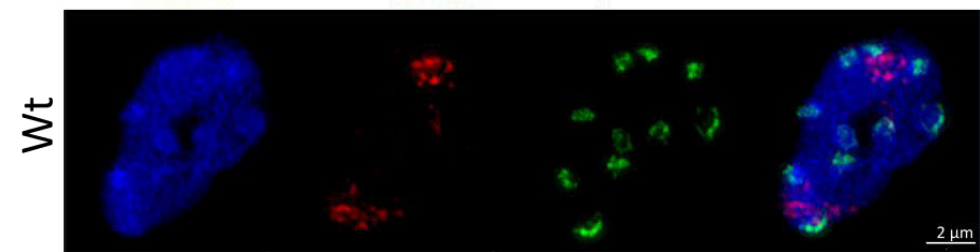

mo

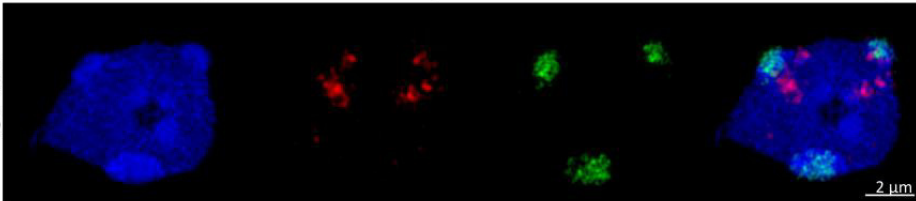

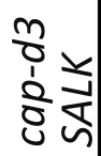

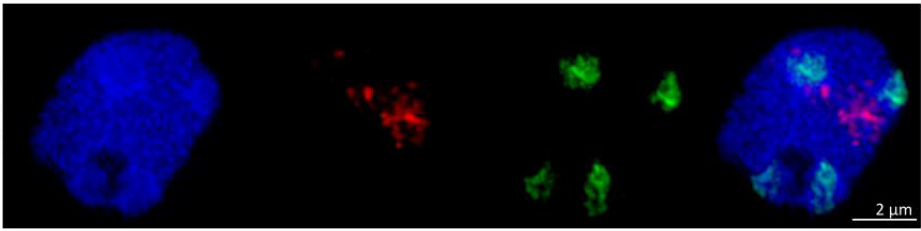

f)

\section{Centromeric association cap-d3 mutants}

$-\underset{(n=53)}{\operatorname{cap}-d 3 S A I L}-\underset{(n=47)}{\operatorname{cap}-d 3 S A L K} \odot \underset{(n=51)}{W \mathrm{t}}$

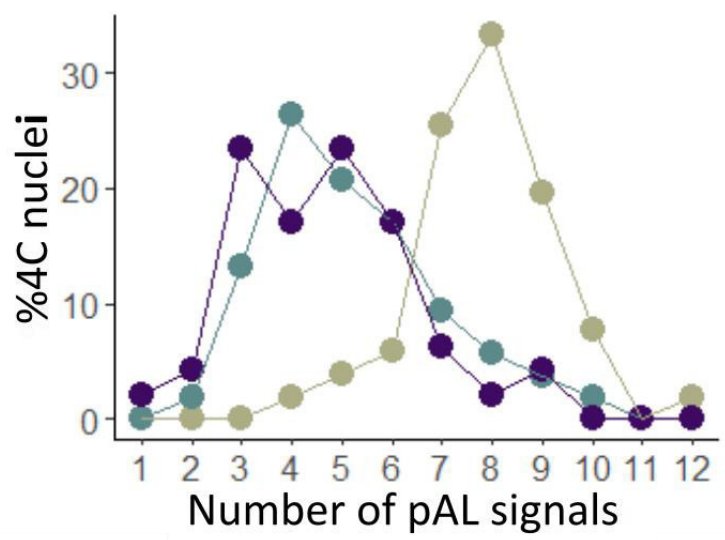

c)

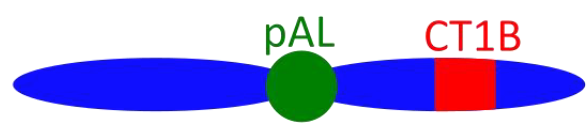

e) Chromosome territory 1 bottom and nuclear area
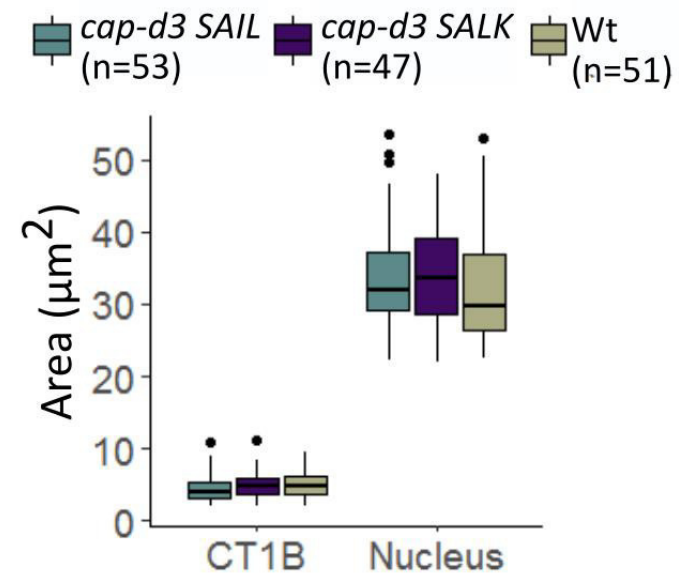
Centromeric association
complemeted line

$-\underset{(n=313)}{c a p-d 3 S A L K} \cdot \underset{(n=315)}{\text { cap-d3 SALK comp }} \cdot \underset{(n=100)}{W t}$

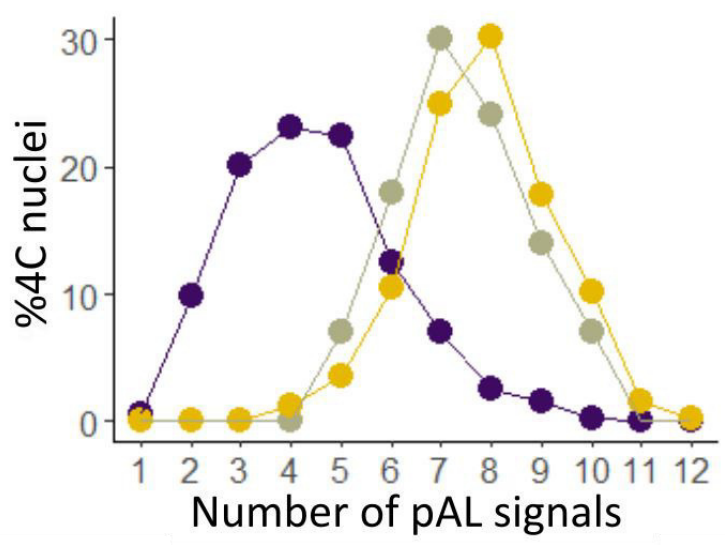

Figure 5. cap-d3 mutations impair centromere distribution, but not CT compaction. a) Gene structure model of CAP-D3. Red boxes represent exons, lines the introns and the lighter red box the 3'UTR. The T-DNA insertion sites of the cap-d3 SAIL and cap-d3 SALK lines are indicated. b) Rosette leave stage phenotypes of homozygous cap-d3 SAIL, cap-d3 SALK and wildtype (Wt) plants. c) Schematic representation of chromosome 1 and the localization of the chromosome territory 1 bottom part (CT1B), and the centromeric pAL probe (labelling all ten centromeres present in the nuclei). d) SIM of a 4C nucleus labelled by FISH with the CT1B and pAL probes. e) Box plot diagram of the CT1B and the nucleus area sizes of cap-d3 SAIL, cap-d3 SALK and Wt nuclei. The boxes indicate upper and lower quartiles and the black bar the median. f) and g) pAL signal frequencies in 4C nuclei of cap-d3 SAIL, cap-d3 SALK, cap-d3 SALK complemented and Wt. $\mathrm{n}=$ total number of nuclei analyzed from two different plants in $\mathbf{e}$ ) and $\mathbf{f}$ ), and from three different plants in $\mathbf{g})$. 
a)

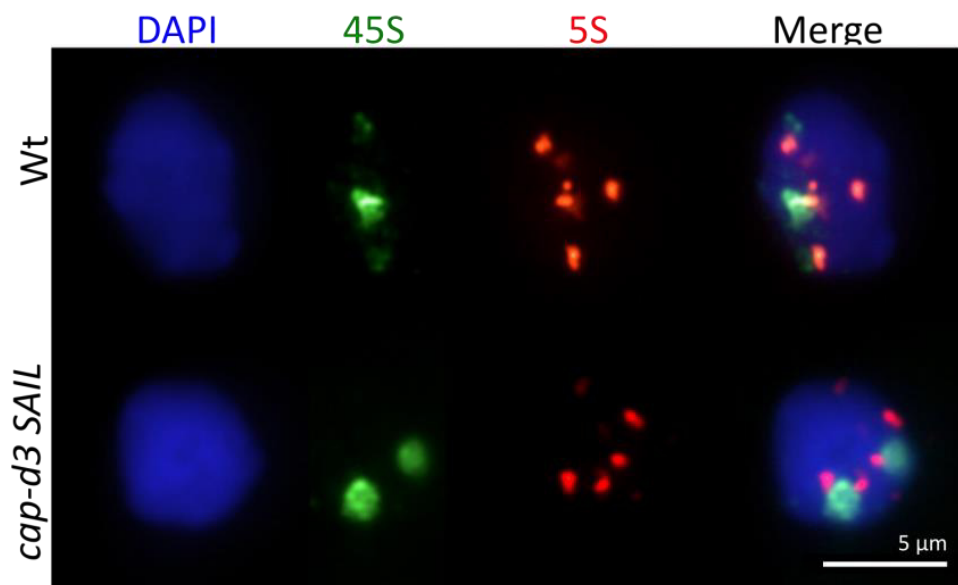

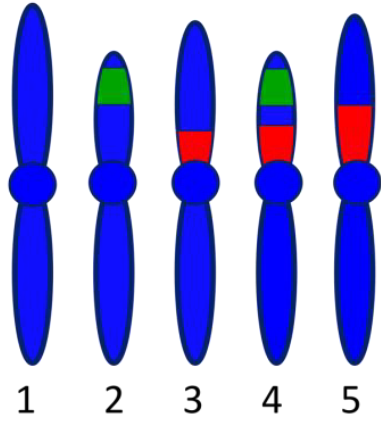

$45 S$ rDNA 5S rDNA b)

\section{Distribution 45S rDNA in cap-d3 mutants}

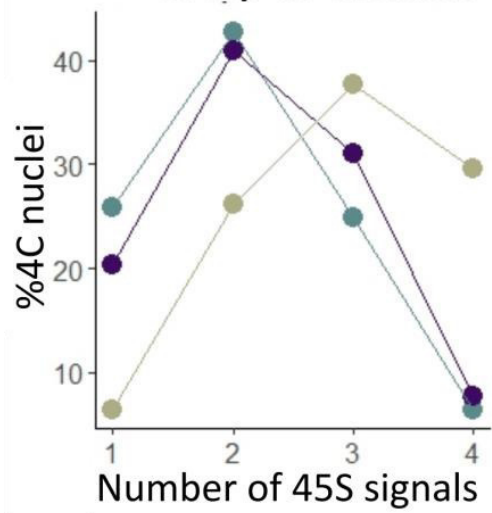

d)

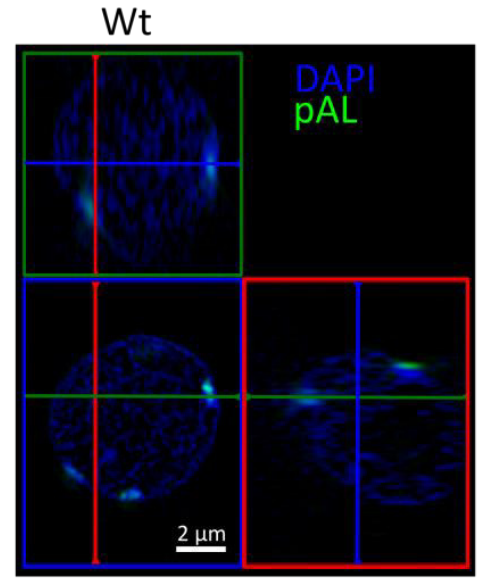

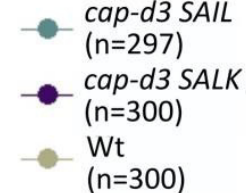

cap-d3 SAIL

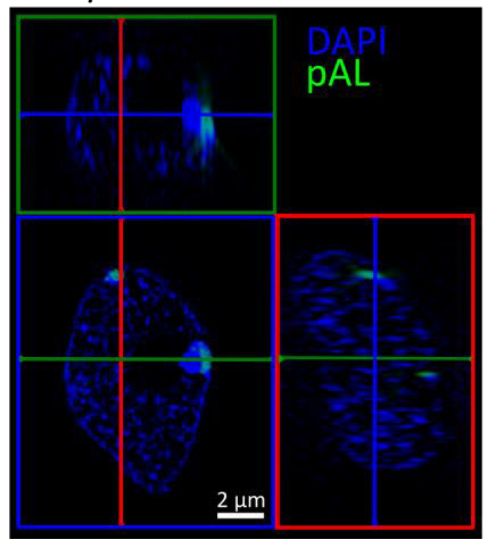

c)

Distribution 45S rDNA in cap-d3 mutants

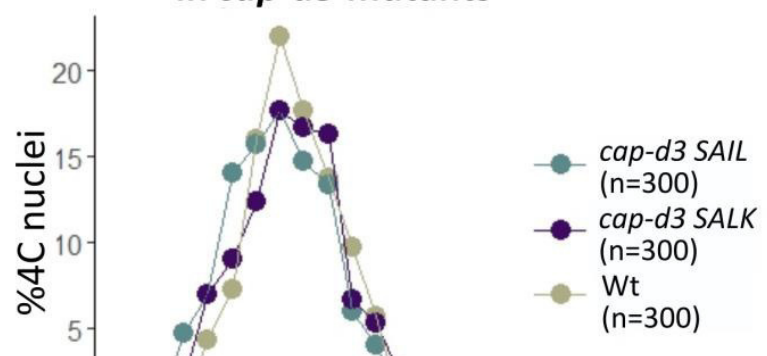

Number of $5 \mathrm{~S}$ signals

cap-d3 SALK

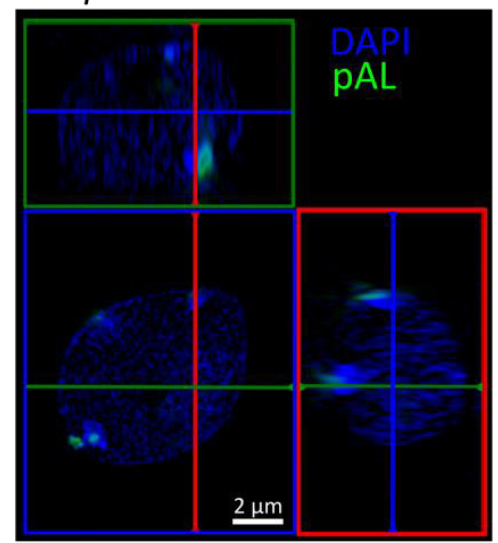

Figure 6. cap-d3 mutations induce of 45S rDNA association, but do not influence 5S rDNA and the spatial centromere arrangement in interphase nuclei. a) FISH signals of 45S rDNA and 5S rDNA on 4C nuclei of wild-type (Wt) and cap-d3 SAIL mutants. The ideogram (right) represents the $A$. thaliana chromosomes, showing the localization of $45 S$ and $5 S$ rDNA. b) and c) Frequency of $45 \mathrm{~S}$ and $5 \mathrm{~S}$ rDNA signals in $4 \mathrm{C}$ nuclei of $\mathrm{Wt}$ and the cap- $\mathrm{d} 3$ mutants. $\mathrm{n}=$ total number of nuclei analyzed from three different plants. d) SIM orthogonal view of FISH with the centromeric repeat (pAL) on structurally preserved acrylamide-embedded nuclei of Wt and cap-d3 mutants. Blue, green and red rectangles show $x-y$, $\mathrm{x}-\mathrm{z}$ and $\mathrm{y}-\mathrm{z}$ optical cross-sections, respectively. 

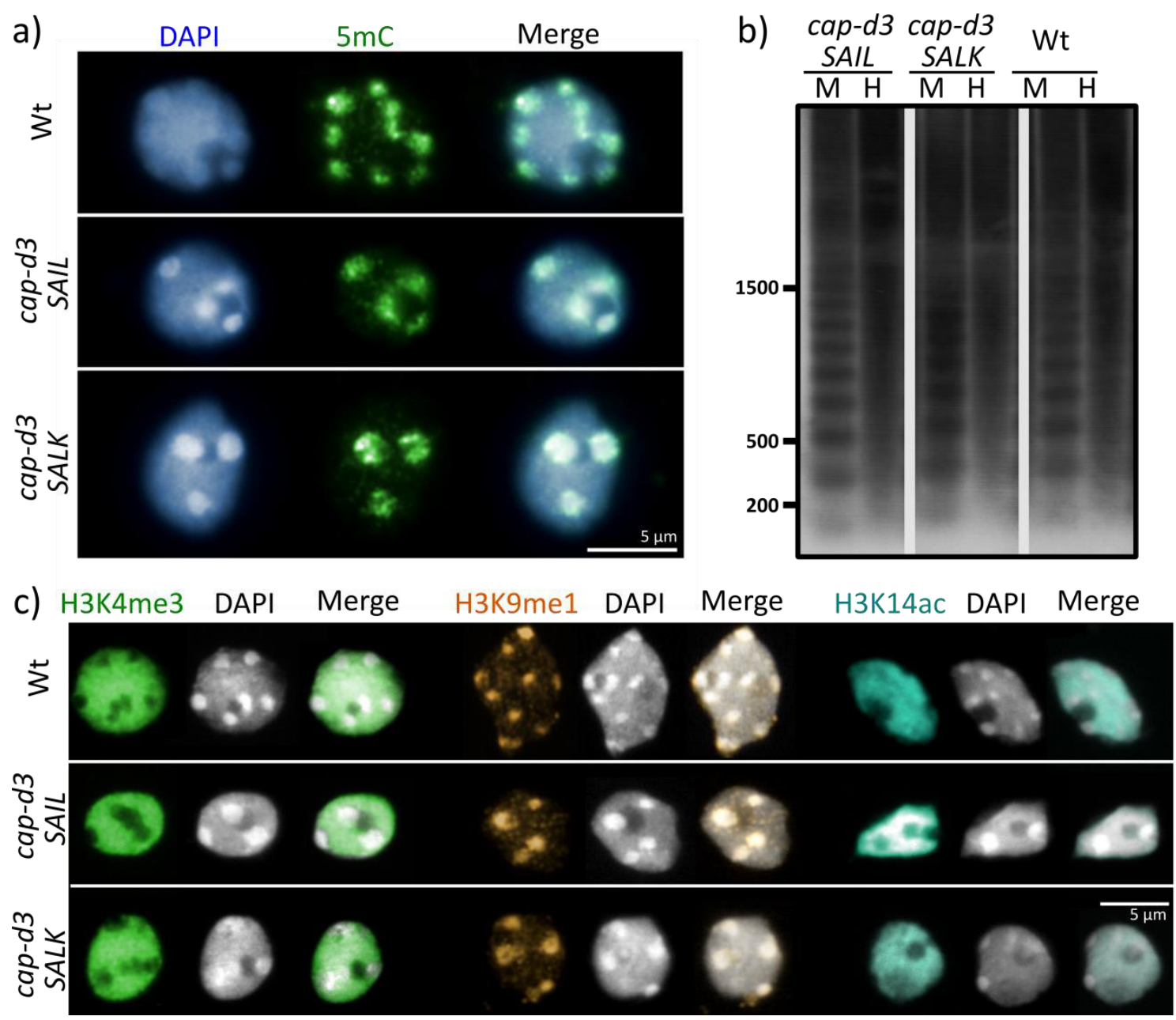

Figure 7. cap-d3 mutations do not modify the epigenetic landscape in interphase nuclei. a) 5-methyl-cytosine immunolocalization on $4 \mathrm{C}$ nuclei of wild-type $(\mathrm{Wt})$ and the cap- $d 3$ mutants. b) Southern blot analysis of the cap- $d 3$ mutants and Wt genomic DNA digested with $\operatorname{HpaII}(\mathrm{H})$ or $M s p I(\mathrm{M})$ and hybridized with the $\mathrm{P}^{32}$-labelled centromeric repeat pAL do not show different digestion patterns. c) Immunolocalization of histone H3K4me3, H3K9me1 and $\mathrm{H} 3 \mathrm{~K} 14 \mathrm{ac}$ on $4 \mathrm{C}$ nuclei of $\mathrm{Wt}$ and the cap- $d 3$ mutants. 
a)
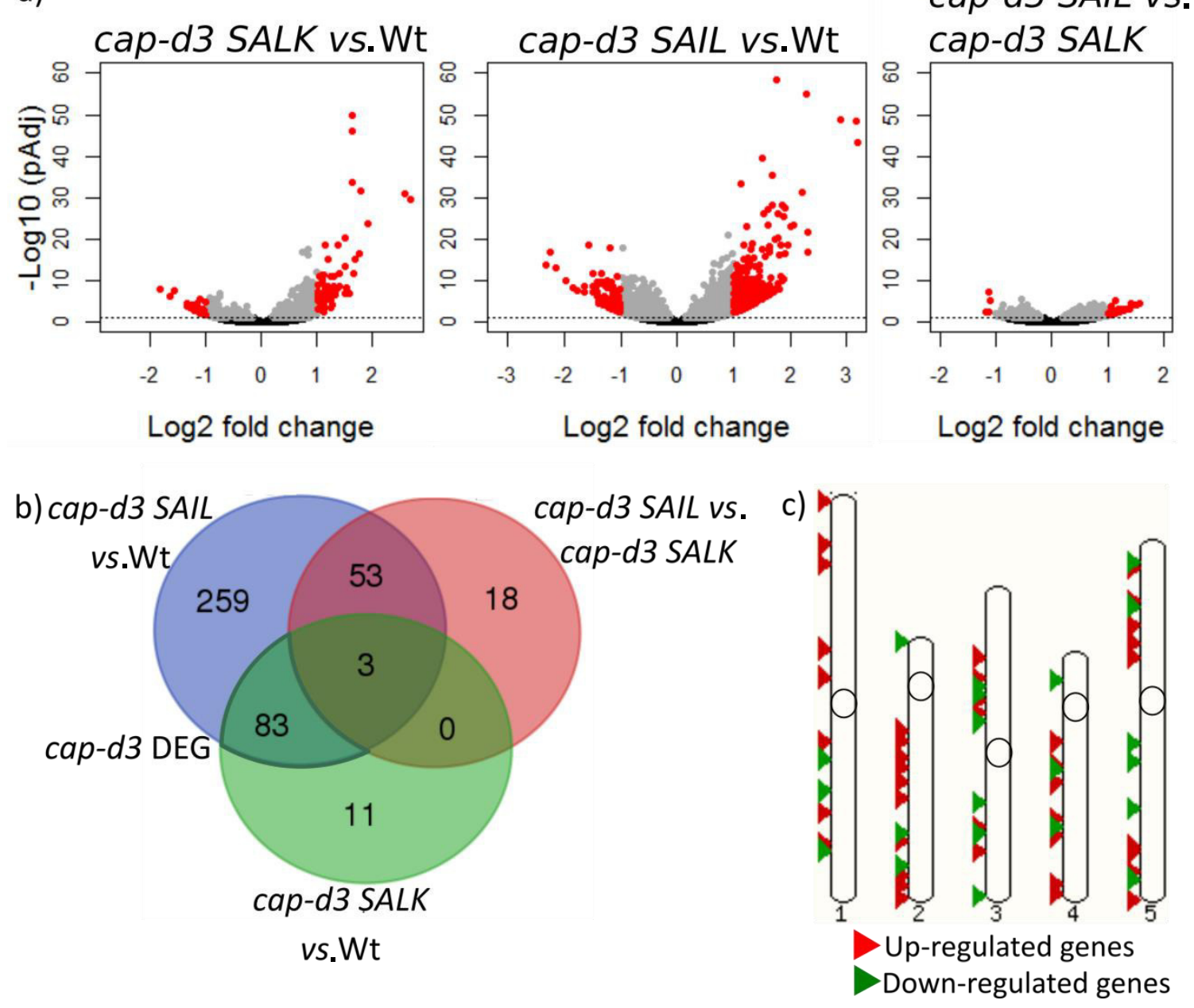

Figure 8. Transcriptome analysis of cap-d3 mutants and wild-type plantlets. a) Volcano plots showing transcriptome comparisons between cap-d3 SALK, cap-d3 SAIL and Wt. The horizontal dotted line corresponds to $\mathrm{pAdj}=0.05$. Genes below are depicted in black and above in grey. The red genes are differentially expressed (DEG) at a threshold of 2 fold change (i.e., up-regulated: $\geq 1 \log 2$ FC, or down-regulated: $\leq-1 \log 2$ FC) and with a pAdj $\leq 0.05$. pAdj is the p-value corrected for multiple testing with the Benjamini-Hochberg adjustment. b) Venn diagram showing the DEG across the three comparisons. Each circle comprises all the DEG genes of one comparison and the intersections between circles are the common DEG. For example: the blue circle represents the cap-d3 SAIL vs. Wt DEG, which are 398, of those: 83 are the same as in cap-d3 SALK vs. Wt, 53 are the same as in cap-d3 SAIL vs. cap-d3 SALK, 3 are differentially expressed in all comparison and 259 are only present in cap-d3 SAIL vs. Wt. c) The ideogram of A. thaliana chromosomes showing the position of the 83 cap-d3 DEG along the chromosomes. 


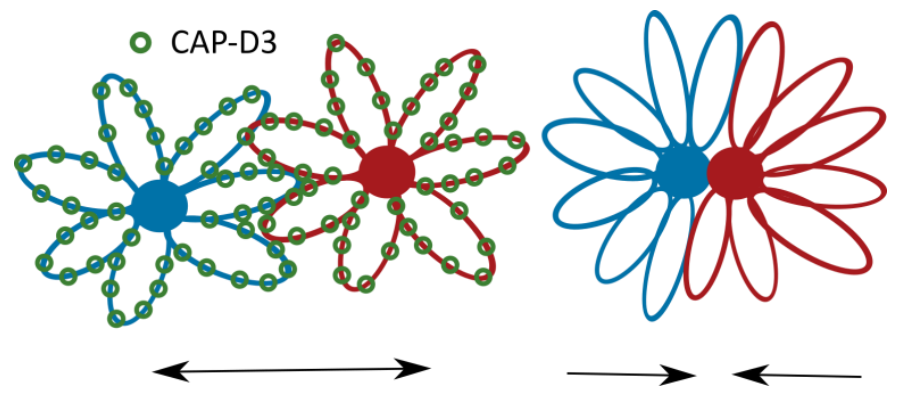

Figure 9. Model explaining the function of Arabidopsis CAP-D3 in interphase nuclei. Two chromosomes are represented in blue and in red with euchromatin emanating loops from their pericentromeric chromocenters (rosette chromosome model; Franz et al., 2002; de Nooijer et al., 2009). CAP-D3 (green circles) localizes along euchromatin creating the chromatin loops rigid to keep the chromocenters separated (left). In absence of CAPD3 the chromatin loops are not stiff enough to counterbalance the depletion-attraction forces (Marenduzzo et al., 2006). Consequently, the chromocenters cluster (right). 
a) CAP-D2

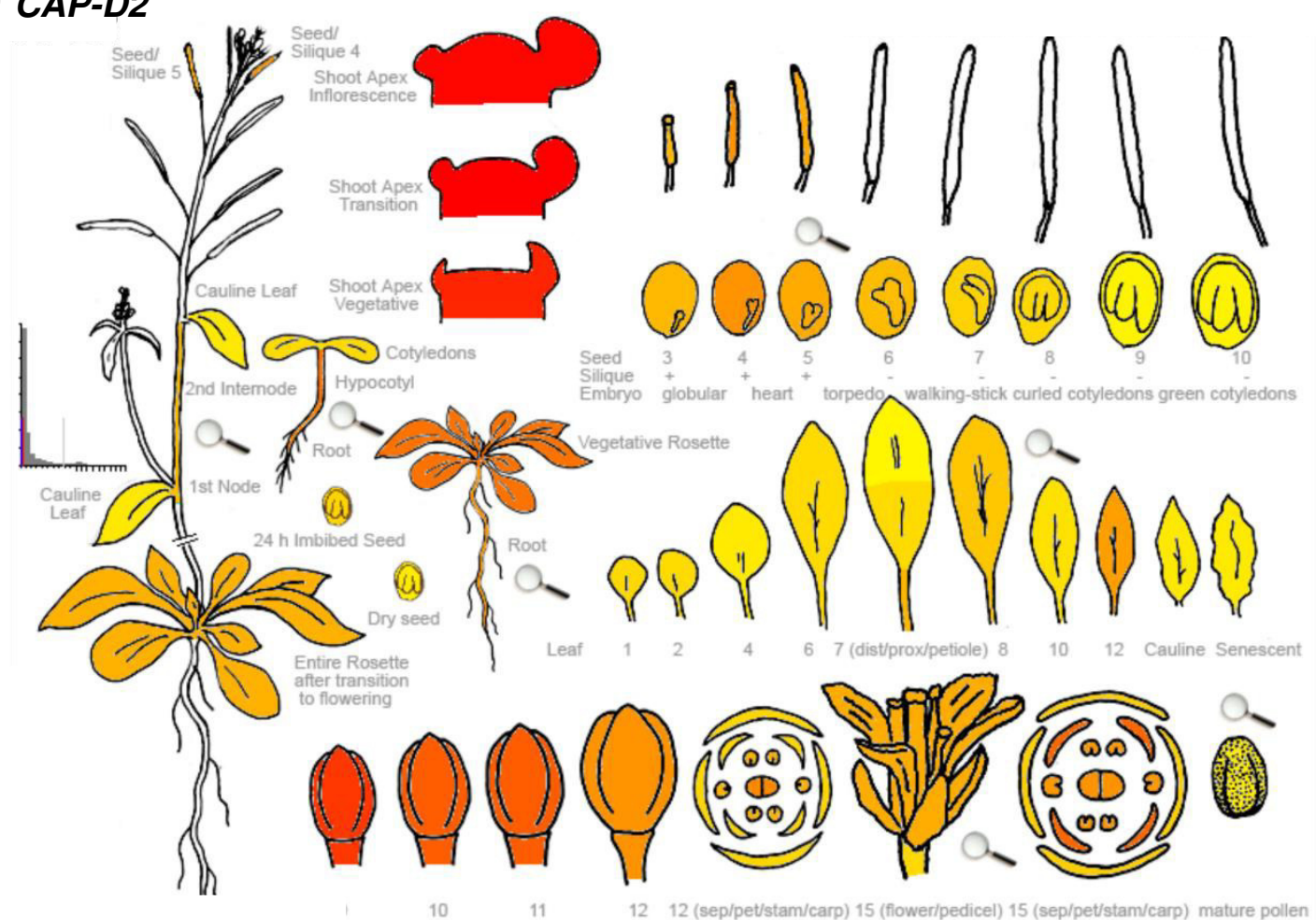

b) $C A P-D 3$

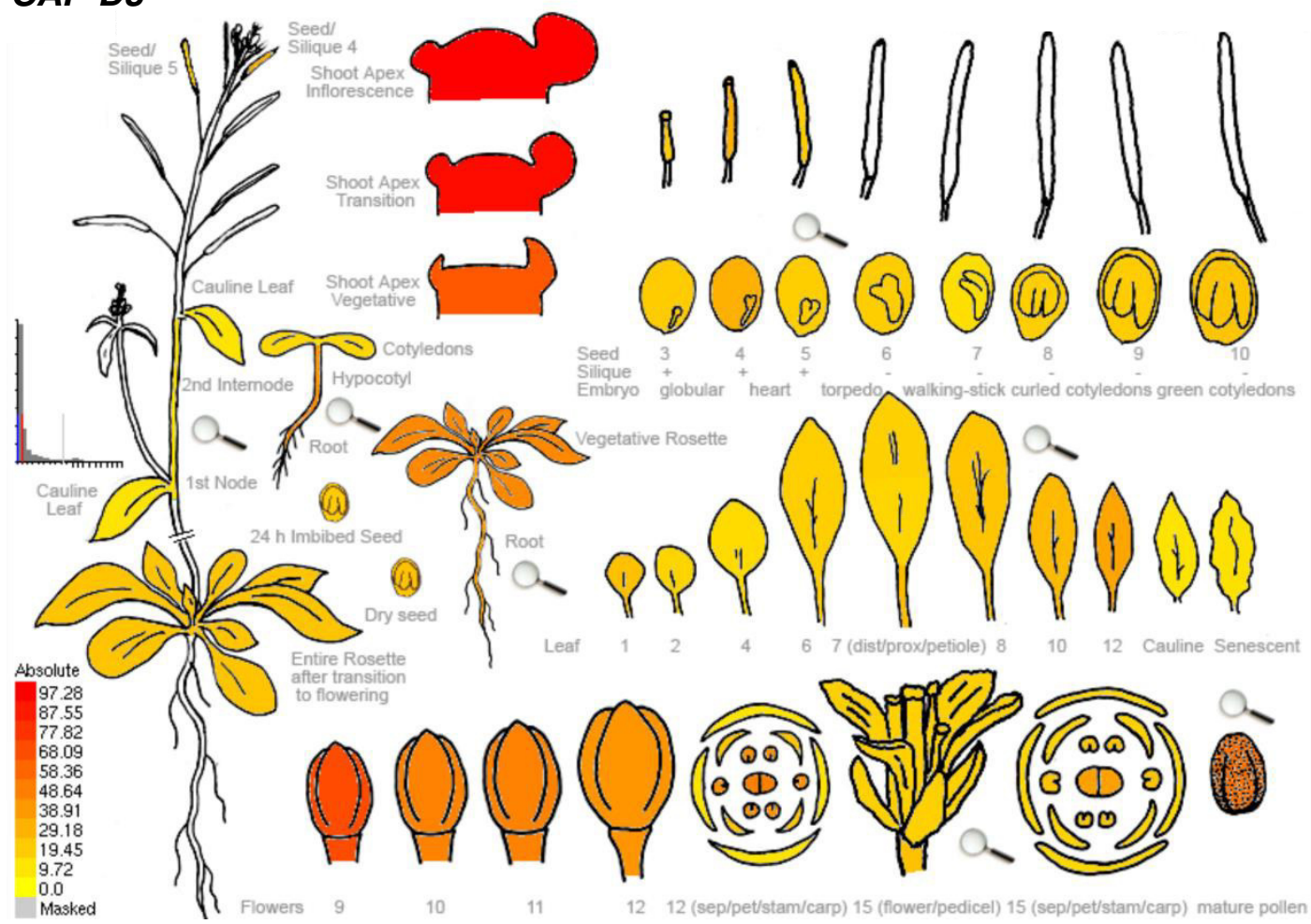

Figure S1. In silico analysis of A. thaliana CAP-D2 and CAP-D3 expression. The results obtained with the Arabidopsis eFP Browser 2.0 (bar.utoronto.ca) revealed a similar expression level for both genes with high (red), medium (orange) and low (yellow) expression in different organs and developmental stages. 


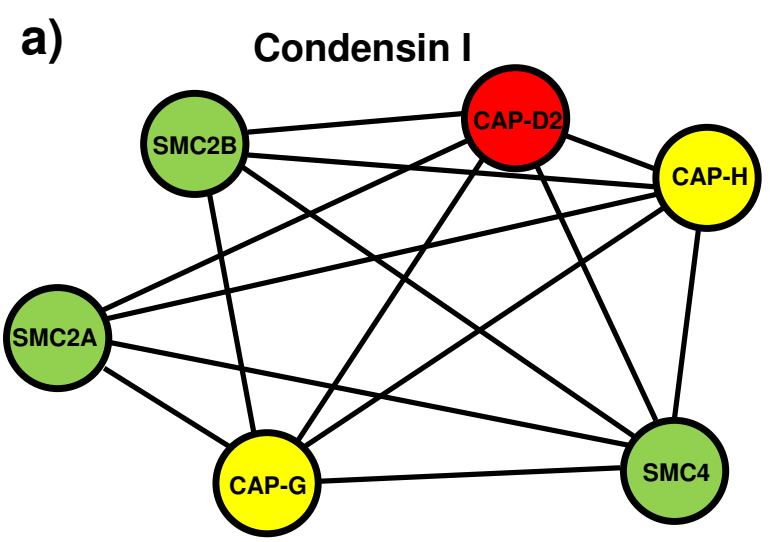

Score $>0.90$ b)

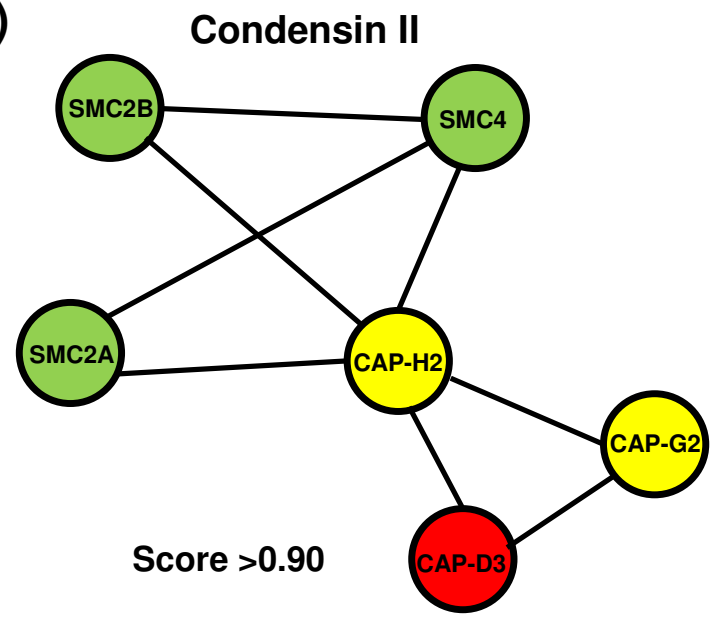

c)

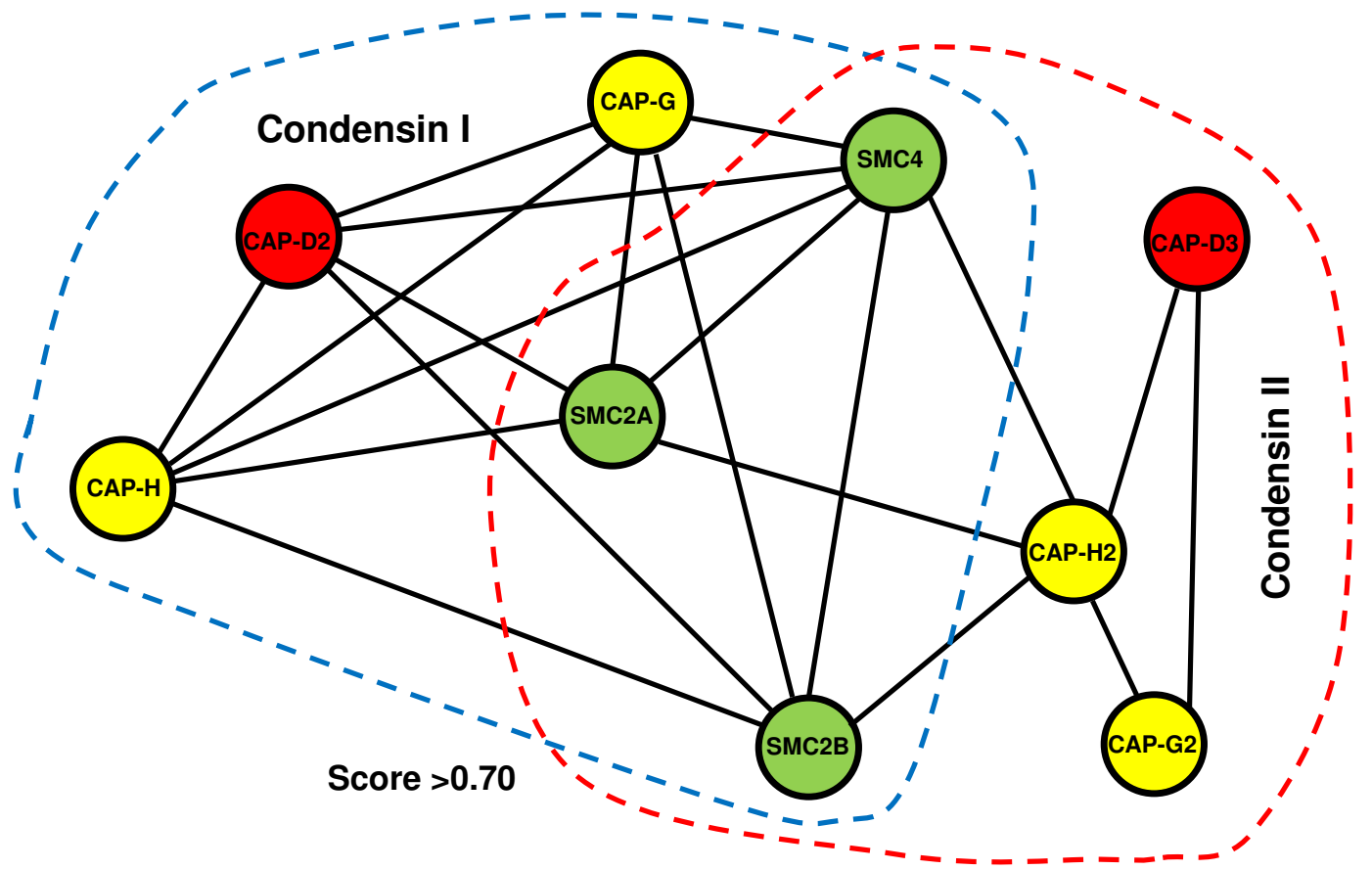

Figure S2. Protein-protein interaction network of CAP-D2 (condensin I) and CAP-D3 (condensin II). Both A. thaliana CAP-D2 (a, c) and CAP-D3 (b, c) proteins (red) interact potentially with the other coiled-coil condensin SMC complex components (green) and the condensin I- and condensin IIspecific subunits (yellow). The network was generated by a STRING program (http://string-db.org/) analysis at scores $>0.90$ (a, b) and $>0.70$ (c), respectively. The black lines in between the proteins indicate the supporting evidence from experimental data available from different species. The dashed lines embrace the condensin I and II subunits in (c). 


\section{CAP-D2-GS CAP-D3-GS}

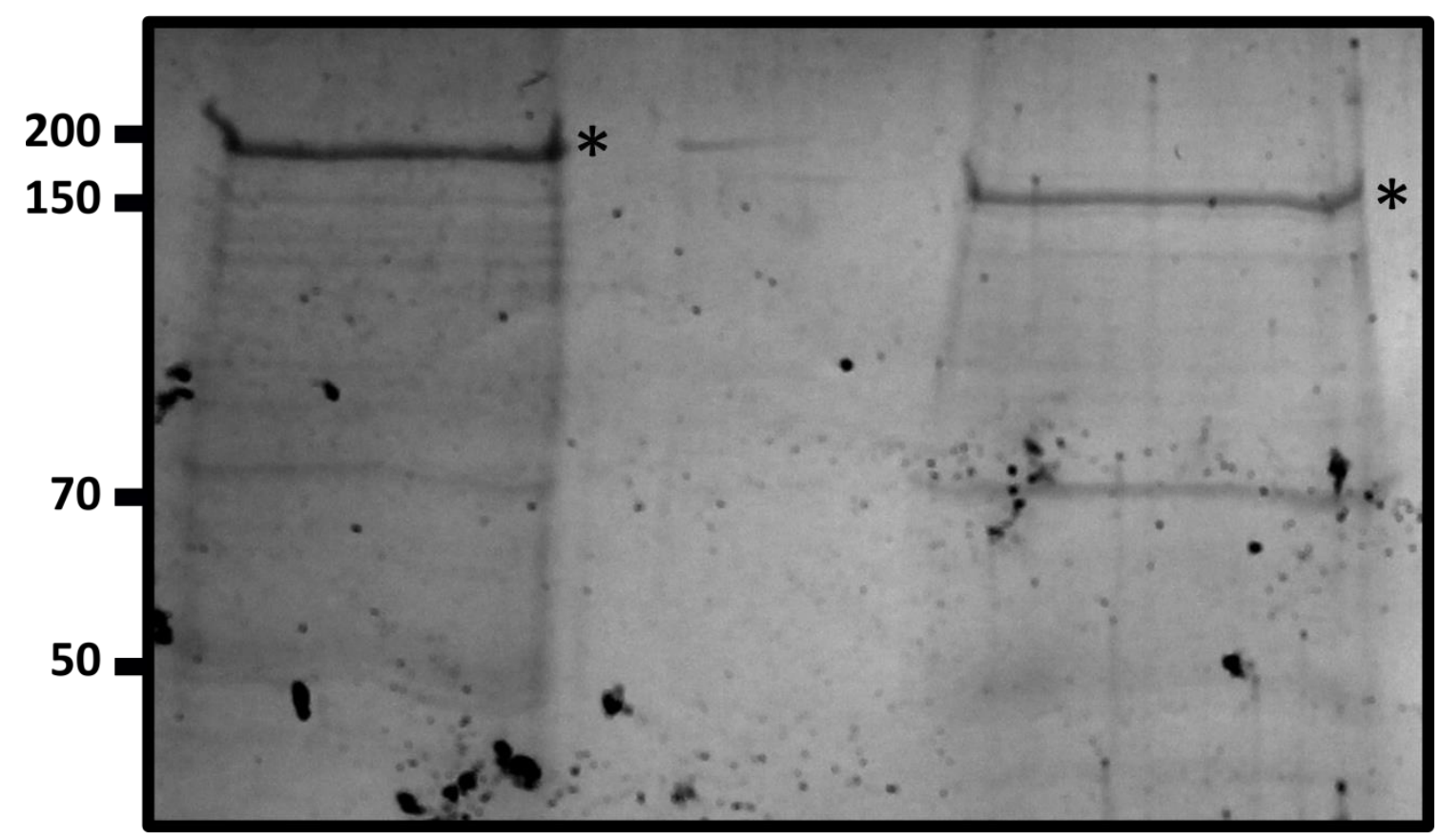

Figure S3. Affinity purified CAP-D2 and CAP-D3 GS-tagged. Coomasie staining of a SDS-PAGE gel with protein extracts from cells expressing CAP-D2-GS and CAP-D3-GS. The asterisks indicate .the CAP-D2GS (176 kDa) and CAP-D3-GS (163 kDa) proteins , respectively. 


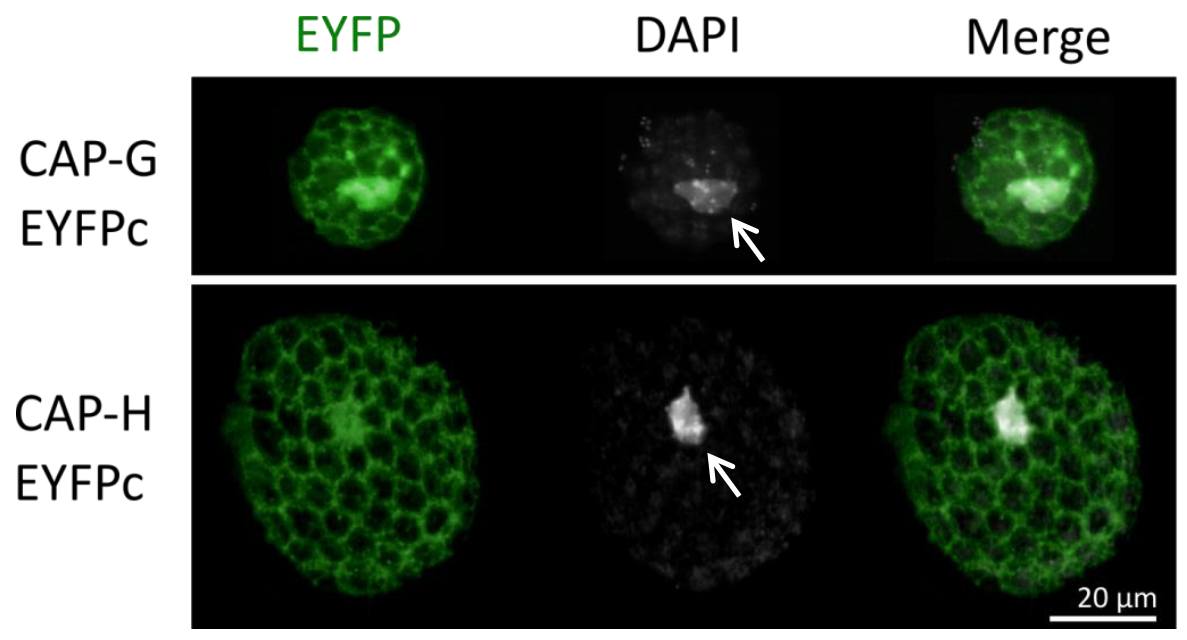

Figure S4. CAP-G and CAP-H fused to EYFPc localize in the nucleus (arrows) and cytoplasm of $A$. thaliana protoplasts. The dark regions are chloroplasts.

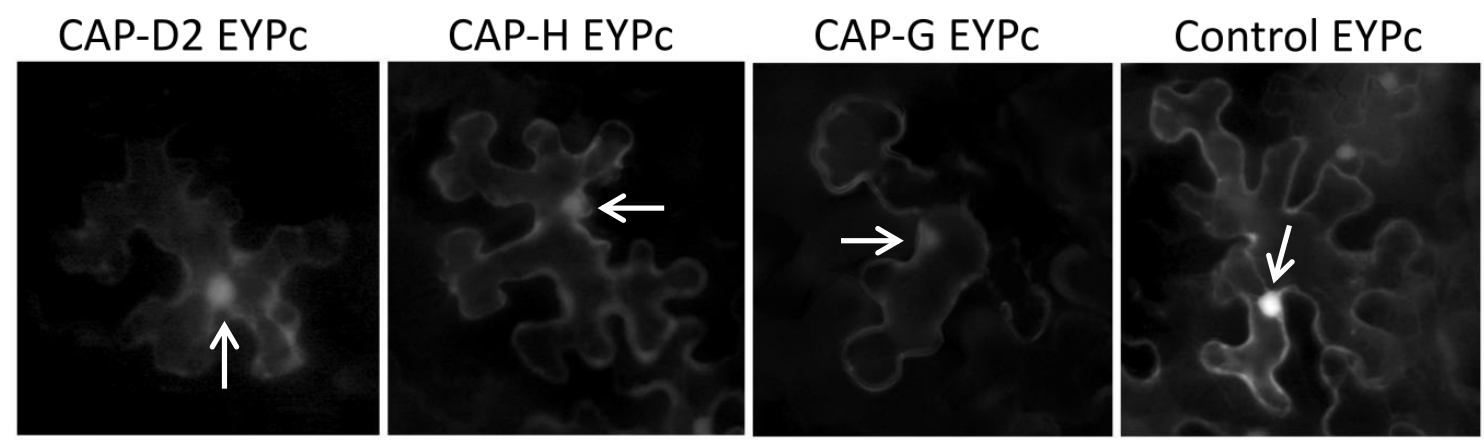

Figure S5. CAP-D2, CAP-H and CAP-G fused to EYFPc localize in the nucleus (arrows) and cytoplasm of $\boldsymbol{N}$. benthamiana leaf epidermal cells. 


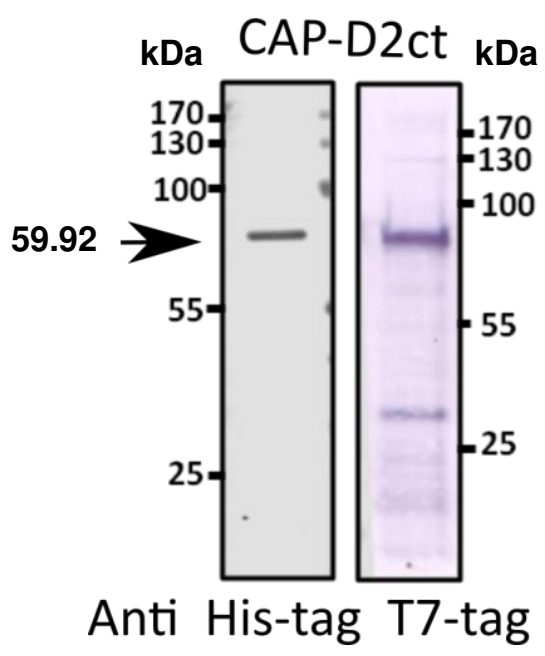

Figure S6. Western blot analysis confirms the correct size of the CAP-D2 recombinant protein (CAP-D2ct). Tested against anti-His-tag and anti-T7-tag the recombinant protein produced in E. coli has a the expected weight of $59.92 \mathrm{kDa}$ including the T7- and His-tags on the N-t and C-termini, respectively. The arrow marks the band containing the CAP-D2_ct recombinant protein.

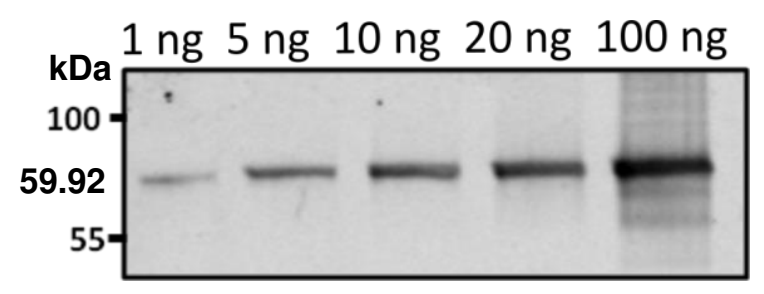

Figure S7. Western blot on different amounts (1-100 ng) of the CAP-D2_ct recombinant protein against the anti-CAP-D2 serum indicates the high sensitivity of anti-CAP-D2. 


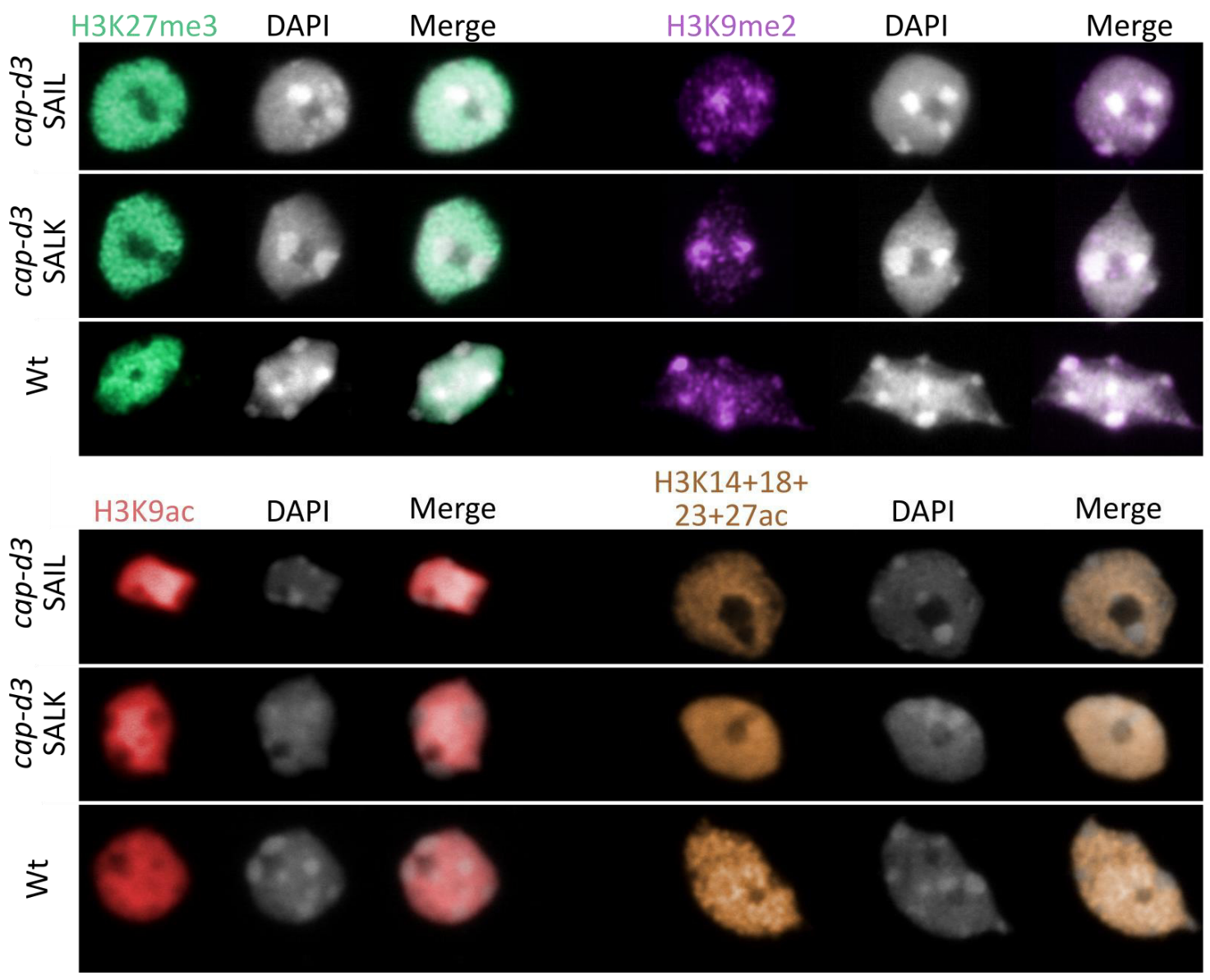

Figure S8. Immunolocalization of histone modifications in cap-d3 mutants and wild-type plants. No differences were detected in $4 \mathrm{C}$ nuclei of wild-type (Wt) and the cap-d3 SAIL, cap-d3 SALK mutants tested with antibodies against histone H3K27me3 (euchromatic); H3K9me2 (heterochromatic); H3K9ac and with antibodies recognizing $\mathrm{H} 3 \mathrm{~K} 14+18+23+27 \mathrm{ac}$. 